\title{
Spectral properties of In II from MCDHF calculations
}

\author{
Per Jönsson ${ }^{1}$ and Martin Andersson ${ }^{2}$ \\ ${ }^{1}$ Nature, Environment, Society, Malmö University, S-205 06 Malmö, Sweden \\ 2 Department of Physics, Lund University, Box 118, S-221 00 Lund, Sweden \\ E-mail: per.jonsson@ts.mah.se
}

Received 19 April 2007, in final form 10 May 2007

Published 5 June 2007

Online at stacks.iop.org/JPhysB/40/2417

\begin{abstract}
We report extensive relativistic multiconfiguration Dirac-Hartree-Fock calculations of oscillator strengths and hyperfine structures for a large number of electric dipole transitions in In II. Results for the $5 \mathrm{~s}^{2}{ }^{1} \mathrm{~S}_{0}-5 \mathrm{~s} 5 \mathrm{p}{ }^{3} \mathrm{P}_{0}^{\mathrm{o}}$ hyperfine induced transition are also presented. Core polarization is accounted for by means of explicit CI. To describe spin-polarization effects configuration state functions obtained by single excitations from all core-shells are included in the expansions. The computed oscillator strength for the $5 \mathrm{~s}^{2}{ }^{1} \mathrm{~S}_{0}-5 \mathrm{~s} 5 \mathrm{p}^{3} \mathrm{P}_{1}^{\mathrm{o}}$ intercombination transition is in good agreement with laser spectroscopy measurements of $\mathrm{In}^{+}$ions in a radio-frequency trap. The calculated magnetic dipole hyperfine interaction constants agree very well with experimental constants derived from Fourier transform spectra. The problem with offdiagonal interactions affecting the hyperfine structures in closely spaced finestructure levels is discussed.
\end{abstract}

\section{Introduction}

There are a number of highly accurate measurements of various spectral properties in In II. New wavelengths, energy levels and hyperfine structure constants for a large number of states have been derived from Fourier transform spectra [1]. The isotope structure of the $5 \mathrm{~s}^{2}{ }^{1} \mathrm{~S}_{0}-5 \mathrm{~s} 5 \mathrm{p}^{3} \mathrm{P}_{1}^{\mathrm{o}}$ line has been studied by means of laser spectroscopy of ions in a radio-frequency trap [2]. In the same experiment the lifetimes of the ${ }^{3} \mathrm{P}_{1}^{\mathrm{o}}$ and ${ }^{3} \mathrm{P}_{0}^{\mathrm{o}}$ states were determined using intensitymodulated laser excitation. The decay of the ${ }^{3} \mathrm{P}_{0}^{\mathrm{o}}$ state is strictly forbidden. However, for atoms with nuclear spin the hyperfine interaction opens decay channels through the admixture of ${ }^{1} \mathrm{P}_{1}^{\mathrm{o}}$ and ${ }^{3} \mathrm{P}_{1}^{\mathrm{o}}$. This effect has been theoretically studied in a number of cases e.g. [3-5], but there are only a few experimental values to compare with. The hyperfine structures of levels in the $5 \mathrm{~s}^{2}{ }^{1} \mathrm{~S}_{0}-5 \mathrm{~s} 5 \mathrm{p}^{3} \mathrm{P}_{1}^{\mathrm{o}}$ and $5 \mathrm{~s} 5 \mathrm{p}^{3} \mathrm{P}_{1,2}^{\mathrm{o}}-5 \mathrm{~s} 6 \mathrm{~s}^{3} \mathrm{~S}_{1}$ transitions were accurately determined by Larkins and Hannaford [6] using a high-resolution scanning echelle monochromator. In addition to these measurements, there are also beam-foil measurements corrected by the arbitrarily normalized decay curve (ANDC) technique of lifetimes for 12 states [7].

During the years there have been several calculations of transition probabilities using different methods. Among the more recent efforts we mention the work by Hibbert [8], 
Migdalek and Baylis [9], Migdalek and Bojara [10], Das and Idrees [11], Chou and Huang [12], Chou et al [13], Lavin and Martin [14], Biémont and Zeippen [15] and Biémont et al [16]. To the knowledge of the authors hyperfine structures and isotope shifts have so far not been theoretically investigated.

Indium is also of astrophysical interest. Indium shows the largest discrepancy between the photospheric and meteoritic values of abundance among all elements with $Z>3$ [17]. The photospheric value is determined from a single In I line at $451.1 \mathrm{~nm}$ in the solar spectrum and one may suspect that unidentified blends cause the discrepancy. No In II lines have been identified in the solar spectrum even though such lines could be expected due to the low ionization energy of In I.

The purpose of the present work is to improve and complement the dataset for In II and to explore the capacity of the grasp2K multiconfiguration Dirac-Hartree-Fock package [18] to predict oscillator strengths and hyperfine structures in 'spectrum calculations' including states high up in the Rydberg series.

\section{Theory}

\section{1. $M C D H F$}

The multiconfiguration Dirac-Hartree-Fock (MCDHF) method [19], as implemented in the grasp2K program package [18], was used in the present work. Starting from the DiracCoulomb Hamiltonian

$$
H_{\mathrm{DC}}=\sum_{i}\left(c \boldsymbol{\alpha}_{i} \cdot \boldsymbol{p}_{i}+\left(\beta_{i}-1\right) c^{2}+V_{i}^{N}\right)+\sum_{i>j} 1 / r_{i j}
$$

where $V^{N}$ is the monopole part of the electron-nucleus Coulomb interaction, the atomic state functions were obtained as linear combinations of symmetry adapted configuration state functions (CSFs)

$$
\left|\Gamma P J M_{J}\right\rangle=\sum_{j=1}^{N} c_{j}\left|\gamma_{j} P J M_{J}\right\rangle .
$$

Here $J$ and $M_{J}$ are the angular quantum numbers and $\mathrm{P}$ is the parity. $\gamma$ denotes other appropriate labelling of the configuration state function, for example orbital occupancy and coupling scheme. Normally the label $\Gamma$ of the atomic state function is the same as the label of the dominating CSF. The configuration state functions were built from products of oneelectron Dirac orbitals. In the relativistic self-consistent field procedure both the radial parts of the Dirac orbitals and the expansion coefficients were optimized to self-consistency. The Breit interaction

$$
B_{i j}=-\frac{1}{2 r_{i j}}\left[\boldsymbol{\alpha}_{i} \cdot \boldsymbol{\alpha}_{j}+\frac{\left(\boldsymbol{\alpha}_{i} \cdot \boldsymbol{r}_{i j}\right)\left(\boldsymbol{\alpha}_{j} \cdot \boldsymbol{r}_{i j}\right)}{r_{i j}^{2}}\right]
$$

was included in subsequent configuration interaction (CI) calculations [20].

\subsection{Isotope shift}

Corrections to the calculated energy structure due to isotope-dependent recoil motion of the nucleus and finite nuclear volume effects were included in first-order perturbation theory with the atomic state functions as zero-order functions. The corrections can be written as

$$
E_{M}=\left(S_{\mathrm{nms}}+S_{\mathrm{sms}}\right) \frac{1}{M}+\frac{2}{3} \pi Z \rho(0)\left\langle r_{M}^{2}\right\rangle
$$


where

$$
S_{\mathrm{nms}}=\left\langle\Gamma P J M_{J}\left|\sum_{i}\left(c \boldsymbol{\alpha}_{i} \cdot \boldsymbol{p}_{i}+\left(\beta_{i}-1\right) c^{2}\right)\right| \Gamma P J M_{J}\right\rangle
$$

and

$$
S_{\mathrm{sms}}=\left\langle\Gamma P J M_{J}\left|\sum_{i<j} \mathbf{p}_{i} \cdot \mathbf{p}_{j}\right| \Gamma P J M_{J}\right\rangle
$$

are, respectively, the normal and specific mass shift parameters and

$$
\rho(0)=\left\langle\Gamma P J M_{J}\left|\sum_{i} \delta\left(\mathbf{r}_{i}\right)\right| \Gamma P J M_{J}\right\rangle
$$

the electron density at the site of the nucleus [21]. $M$ is the mass of the nucleus and $\left\langle r_{M}^{2}\right\rangle$ is the root-mean-square radius, both in atomic units. From these quantities, the transition isotope shift $\Delta E_{M^{\prime}, M}$ between two isotopes $M^{\prime}$ and $M$ was obtained as

$\Delta E_{M^{\prime}, M}=\left(\Delta S_{\mathrm{nms}}+\Delta S_{\mathrm{sms}}\right)\left(\frac{1}{M^{\prime}}-\frac{1}{M}\right)+\frac{2 \pi}{3} Z \Delta \rho(0)\left(\left\langle r_{M^{\prime}}^{2}\right\rangle-\left\langle r_{M}^{2}\right\rangle\right)$,

where the differences in the electronic quantities are between the values of upper and lower levels in the transition.

\subsection{Hyperfine structure and Landé factors}

The two stable isotopes of indium, ${ }^{115} \operatorname{In}(95.7 \%)$ and ${ }^{113} \operatorname{In}(4.3 \%)$, both have nuclear spin $I=9 / 2$ and the fine-structure levels are split into closely spaced hyperfine levels. The splittings of the fine-structure levels are to first order given by the magnetic dipole $A_{J}$ and electric quadrupole $B_{J}$ hyperfine interaction constants:

$$
\begin{aligned}
& A_{J}=\frac{\mu_{I}}{I} \frac{1}{\sqrt{J(J+1)(2 J+1)}}\left\langle\Gamma P J\left\|\sum_{j}-\mathrm{i} \sqrt{2} \alpha r_{j}^{-2}\left(\alpha_{j} \mathbf{C}^{(1)}(j)\right)^{(1)}\right\| \Gamma P J\right\rangle, \\
& B_{J}=2 Q \sqrt{\frac{J(2 J-1)}{(J+1)(2 J+1)(2 J+3)}}\left\langle\Gamma P J\left\|\sum_{j}-r_{j}^{-3} \mathbf{C}^{(2)}(j)\right\| \Gamma P J\right\rangle .
\end{aligned}
$$

The hyperfine levels of closely spaced fine-structure levels are also affected by the off-diagonal hyperfine interaction [22]. The hyperfine interaction constants as well as the Landé $g_{J}$-factors

$g_{J}=\frac{2}{\sqrt{J(J+1)(2 J+1)}}\left\langle\Gamma P J\left\|\sum_{j}-\mathrm{i} \frac{\sqrt{2}}{2 \alpha^{2}} r_{j}\left(\boldsymbol{\alpha}_{j} \mathbf{C}^{(1)}(j)\right)^{(1)}+\frac{g_{s}-2}{2} \beta_{j} \boldsymbol{\Sigma}_{j}\right\| \Gamma P J\right\rangle$

were calculated using the Hfszeeman program [23, 24]. The nuclear magnetic dipole moment $\mu_{I}$ and the nuclear quadrupole moment $Q$ were taken from a recent compilation by Stone [25] and we use the values $\mu_{I}=5.5408 \mu_{N}$ and $Q=0.81(5)$ barns for ${ }^{115} \mathrm{In}$.

\subsection{Transition parameters}

The transition parameters, such as weighted oscillator strengths, for multipole transitions between two atomic states $\Gamma P J M_{J}$ and $\Gamma^{\prime} P^{\prime} J^{\prime} M_{J}^{\prime}$ can be expressed in terms of the reduced transition matrix element

$$
\left\langle\Gamma P J\left\|\mathbf{Q}_{k}^{(\lambda)}\right\| \Gamma^{\prime} P^{\prime} J^{\prime}\right\rangle,
$$


where $Q_{k q}^{(\lambda)}$ is the electromagnetic multipole operator of order $k$ in Coulomb or Babushkin gauge [26]. The superscript designates the type of multipole: $\lambda=1$ for electric multipoles and $\lambda=0$ for magnetic multipoles. To compute the transition matrix element between two atomic state functions described by independently optimized orbital sets, biorthogonal transformations of the atomic state functions were performed [27]. In the new representation, the evaluation of the matrix element was done using standard Racah-algebra techniques.

\section{Calculations}

\subsection{Spectrum}

It is, from some perspectives, desirable to perform separate calculations for each of the studied atomic states. This approach, however, is impractical and time consuming when considering large portions of a spectrum. In this work, the atomic state functions were instead determined simultaneously in extended optimal level (EOL) calculations on the weighted energy average of the states [28]. As a starting point two MCDHF calculations were performed in the EOL scheme: one calculation with $24 \mathrm{CSFs}$ describing each of the even parity states up to $5 \mathrm{~s} 7 \mathrm{~d}^{1} \mathrm{D}_{2}$, and one calculation with $20 \mathrm{CSFs}$ describing each of the odd parity states up to $5 \mathrm{~s} 5 \mathrm{f}^{1} \mathrm{~F}_{3}^{\mathrm{o}}$. These calculation were followed by calculations with expansions including CSFs obtained by single(S) and double(D) excitations from, respectively, the studied even and odd state reference configurations to active sets of orbitals. Of the excitations from the even and odd state reference configurations at most one was allowed from the outer $4 \mathrm{~d}$ core-shell. The active sets for the even and odd parity states consisting of spectroscopic orbitals from the initial MCDHF calculations were consecutively enlarged by adding three layers of correlation orbitals. Each layer contained orbitals with s, p-, p, d-, d, f-, f symmetries. For the even states one layer also included orbitals with g-, g symmetries. For the odd states two layers included orbitals with g-, g symmetries and one layer contained orbitals with h-, h symmetries. Due to stability problems in the relativistic self-consistent field procedure only the outermost layers of orbitals could be optimized each time. The largest calculation for the even parity states included 49 relativistic orbitals and around 157000 CSFs. The largest calculation for the odd parity states was based on 52 relativistic orbitals and more than 235000 CSFs. All calculations were done on an ordinary PC under Linux.

The MCDHF calculations were followed by relativistic CI calculations including Breit and some QED effects. To account for spin-polarization which is important for the hyperfine interaction constants [29], additional CI calculations were performed. The expansions for these CI calculations were obtained by augmenting the largest expansion from the previous CI calculations with CSFs generated by allowing single excitations from all shells of the initial reference configurations to the active set of orbitals.

\subsection{Ground and first excited states}

In EOL calculations, the included correlation orbitals correct a number of states at the same time. Obviously this will give less accurate results compared to the case where the correlation orbitals are obtained in separate calculations for each of the states. To check the accuracy of the spectrum calculations described above, separate calculations were performed for the $5 \mathrm{~s}^{2}{ }^{1} \mathrm{~S}_{0}$ ground state and the excited $5 \mathrm{~s} 5 \mathrm{p}^{3} \mathrm{P}_{0,1,2}^{\mathrm{o}},{ }^{1} \mathrm{P}_{1}^{\mathrm{o}}$ states. The expansions for the ground state were obtained by allowing SD-excitations from the $5 \mathrm{~s}^{2}, 5 \mathrm{p}^{2}$ and $5 \mathrm{~d}^{2}$ complex to an active set. As before at most one excitation was allowed from the outer $4 \mathrm{~d}$ core-shell. The $5 \mathrm{~s} 5 \mathrm{p}^{3} \mathrm{P}_{0,1,2}^{\mathrm{o}},{ }^{1} \mathrm{P}_{1}^{\mathrm{o}}$ states were treated together in an EOL scheme. The expansions for these states were obtained 
by allowing SD-excitations from the $5 \mathrm{~s} 5 \mathrm{p}$ and $5 \mathrm{p} 5 \mathrm{~d}$ complex to an active set. The active sets were increased by adding five layers of correlation orbitals. All calculations were followed by relativistic CI calculations including Breit and some QED effects. To account for spinpolarization additional $\mathrm{CI}$ calculations were again performed with the expansions from the previous CI calculations augmented by CSFs generated by allowing single excitations from all shells of the initial reference configurations to the active set of orbitals.

\section{Results and discussion}

\subsection{Spectrum properties}

The energy values from the different spectrum calculations are displayed in table 1 . The inclusion of the three layers of correlation orbitals improves the initial energies dramatically. Although the Breit and QED effects in the CI calculations slightly act in the wrong direction, the final energies are in very good agreement with experiment. All levels except $5 \mathrm{p}^{2}{ }^{1} \mathrm{~S}_{0}$ are a few hundred $\mathrm{cm}^{-1}$ too high relative to the ground level indicating that less correlation has been captured for the latter. In table 2 calculated hyperfine structure constants from the CI wavefunctions including spin-polarization are compared with experimental values of Karlsson and Litzén derived from Fourier transform spectra [1]. The calculated and experimentally determined $A$ factors are in very good agreement. For the $B$ factors the situation is different and in many cases there are substantial differences between theory and experiment. In the paper by Karlsson and Litzén it is, however, pointed out that for some levels different transitions did not give quite consistent results for the $B$ factors and the given values, except for the lowest configurations, should therefore mainly be considered as parameters useful together with the $A$ values for describing the observed structure in a model accounting only for diagonal hyperfine interaction. Details of the fitting procedures can be found in the thesis by Karlsson [30]. The large differences between the calculated and experimentally determined $B$ factors indicate that the neglected off-diagonal hyperfine interactions are indeed important for some of the levels. The off-diagonal interaction shifts the positions of the hyperfine levels and, more important, leads to a redistribution of the oscillator strength that modifies the line profiles. The effect of the off-diagonal interaction has been investigated for the $4 \mathrm{~s} 4 \mathrm{~d}^{3} \mathrm{D}-4 \mathrm{~s} 4 \mathrm{f}^{3} \mathrm{~F}^{\circ}$ transition in the homologous Ga II [22] and a similar study of the unanalysed lines of the $5 \mathrm{~s} 5 \mathrm{~d}^{3} \mathrm{D}-5 \mathrm{~s} 4 \mathrm{f}^{3} \mathrm{~F}^{\mathrm{o}}$ transition in In II is in progress [31]. In the last two columns of table 2 the calculated Landé $g_{J}$ values are compared with values assuming pure $L S$ coupling. There is a close agreement between the two sets of $g_{J}$ values although one sees that term mixing in $5 \mathrm{~s} n \mathrm{p}{ }^{1,3} \mathrm{P}_{1}$ becomes more important higher up in the Rydberg series, gradually changing the $g_{J}$ values away from the $L S$ limit.

Calculated oscillator strengths in the Coulomb or Babushkin gauges from the CI wavefunctions are presented in table 3. To save space only transitions with $g f>10^{-3}$ are included. The agreement between the two gauges is very good. All values are completely $a b$ initio and for some transitions with small transition energies the calculated values may be improved by scaling the $g f$ values with the experimental transition energies. In table 4 the present values are compared with experiment and selected values from the semi-empirical HFR calculations by Biémont and Zeippen [15]. The values from the two calculations are in good agreement, the value for the weak $5 \mathrm{~s}^{2}{ }^{1} \mathrm{~S}_{0}-5 \mathrm{~s} 6 \mathrm{p}{ }^{1} \mathrm{P}_{1}^{\mathrm{o}}$ transition being an exception. For this transition the difference between the two gauges is also large, indicating difficulties of some sort. A survey of previous calculations can be found in table 5 of [15]. 
Table 1. Calculated and experimental energy levels in $\mathrm{cm}^{-1}$. Column 1 displays energies from calculations where the configuration expansion were generated by excitations to the initial spectroscopic orbitals. Columns 2-4 show energies from calculations where the active set consisting of the spectroscopic orbitals were enlarged by, respectively, one, two and three layers of correlation orbitals. Column 5 shows the final energy values from the CI calculations including Breit and leading QED effects.

\begin{tabular}{|c|c|c|c|c|c|c|}
\hline Level & $\begin{array}{l}\text { Spectroscopic } \\
\text { orbitals }\end{array}$ & Corr. 1 & Corr. 2 & Corr. 3 & CI & Experimental \\
\hline $5 s^{2}{ }^{1} S_{0}$ & 0.000 & 0.000 & 0.000 & 0.000 & 0.000 & 0.000 \\
\hline $5 s 5 p^{3} P_{0}^{o}$ & 36519.60 & 41292.83 & 42195.51 & 42164.18 & 42084.92 & 42275.997 \\
\hline $5 \mathrm{~s} 5 \mathrm{p}^{3} \mathrm{P}_{1}^{\mathrm{o}}$ & 37588.00 & 42371.49 & 43262.85 & 43232.97 & 43141.86 & 43350.583 \\
\hline $5 \mathrm{~s} 5 \mathrm{p}^{3} \mathrm{P}_{2}^{\mathrm{o}}$ & 39867.69 & 44823.79 & 45715.42 & 45696.12 & 45575.11 & 45829.257 \\
\hline $5 \mathrm{~s} 5 \mathrm{p}^{1} \mathrm{P}_{1}^{\mathrm{o}}$ & 65526.23 & 63612.94 & 63044.28 & 62907.36 & 62779.58 & 63038.534 \\
\hline $5 \mathrm{~s} 6 \mathrm{~s}^{3} \mathrm{~S}_{1}$ & 85766.18 & 92854.78 & 93930.45 & 93791.42 & 93651.19 & 93923.883 \\
\hline $5 s 6 s{ }^{1} S_{0}$ & 90391.54 & 96055.62 & 97071.36 & 96981.07 & 96841.35 & 97030.201 \\
\hline $5 \mathrm{~s} 5 \mathrm{~d}^{1} \mathrm{D}_{2}$ & 87470.27 & 97393.20 & 97879.05 & 97717.15 & 97527.55 & 97628.421 \\
\hline $5 p^{23} P_{0}$ & 94994.22 & 100684.72 & 101392.41 & 101386.10 & 101181.84 & 101607.7 \\
\hline $5 s 5 d^{3} D_{1}$ & 93870.75 & 101754.01 & 102377.13 & 101956.81 & 101791.86 & 102088.975 \\
\hline $5 s 5 d^{3} D_{2}$ & 93942.86 & 101839.70 & 102464.33 & 102044.53 & 101877.06 & 102174.924 \\
\hline $5 s 5 d^{3} D_{3}$ & 94056.44 & 101964.08 & 102595.13 & 102179.22 & 102007.98 & 102308.387 \\
\hline $5 p^{23} \mathrm{P}_{1}$ & 96220.86 & 102225.83 & 103013.72 & 103005.72 & 102777.42 & 103248.84 \\
\hline $5 p^{2}{ }^{3} \mathrm{P}_{2}$ & 98310.97 & 104543.41 & 105358.30 & 105331.00 & 105078.02 & 105565.275 \\
\hline $5 \mathrm{~s} 6 \mathrm{p}^{3} \mathrm{P}_{0}^{\mathrm{o}}$ & 98570.47 & 106042.40 & 107620.57 & 107357.57 & 107215.95 & 107662.708 \\
\hline $5 \mathrm{~s} 6 \mathrm{p}^{3} \mathrm{P}_{1}^{\mathrm{o}}$ & 98775.17 & 106225.84 & 107809.24 & 107538.71 & 107395.76 & 107841.992 \\
\hline $5 \mathrm{~s} 6 \mathrm{p}^{3} \mathrm{P}_{2}^{\mathrm{o}}$ & 99266.96 & 106761.15 & 108398.07 & 108124.32 & 107973.87 & 108430.336 \\
\hline $5 \mathrm{~s} 6 \mathrm{p}^{1} \mathrm{P}_{1}^{\mathrm{o}}$ & 103101.40 & 108552.52 & 109975.04 & 109546.86 & 109401.41 & 109780.223 \\
\hline $5 \mathrm{p}^{21} \mathrm{D}_{2}$ & 108433.85 & 114632.02 & 114706.85 & 114003.51 & 113791.25 & 113884.903 \\
\hline $5 \mathrm{p}^{2}{ }^{1} \mathrm{~S}_{0}$ & 122885.97 & 120500.03 & 121550.50 & 121555.39 & 121364.45 & 121289.09 \\
\hline $5 s 7 s^{3} S_{1}$ & 111677.79 & 119811.43 & 121305.91 & 121362.58 & 121206.33 & 121442.542 \\
\hline $5 \mathrm{~s} 7 \mathrm{~s}{ }^{1} \mathrm{~S}_{0}$ & 113397.06 & 124453.17 & 123770.64 & 123589.69 & 123208.79 & 123372.851 \\
\hline $5 \mathrm{~s} 4 \mathrm{f}^{3} \mathrm{~F}_{2}^{\mathrm{o}}$ & 113858.17 & 121913.41 & 123378.67 & 123372.00 & 123211.89 & 123643.132 \\
\hline $5 \mathrm{~s} 4 \mathrm{f}^{3} \mathrm{~F}_{3}^{\mathrm{o}}$ & 113856.93 & 121911.23 & 123378.67 & 123375.18 & 123220.92 & 123648.050 \\
\hline $5 \mathrm{~s} 4 \mathrm{f}^{3} \mathrm{~F}_{4}^{\mathrm{o}}$ & 113855.29 & 121908.54 & 123389.00 & 123384.36 & 123263.56 & 123664.787 \\
\hline $5 \mathrm{~s} 4 \mathrm{f}^{1} \mathrm{~F}_{3}^{\mathrm{o}}$ & 114110.21 & 122119.00 & 123405.15 & 123426.91 & 123376.22 & 123699.154 \\
\hline $5 s 6 d^{3} D_{1}$ & 114915.61 & 123310.86 & 124629.89 & 124661.88 & 124496.62 & 124742.729 \\
\hline $5 s 6 d^{3} D_{2}$ & 114947.35 & 123344.87 & 124664.17 & 124694.04 & 124527.77 & 124776.712 \\
\hline $5 s 6 d^{3} D_{3}$ & 114995.25 & 123396.95 & 124717.44 & 124744.44 & 124576.64 & 124830.174 \\
\hline $5 \mathrm{~s} 6 \mathrm{~d}^{1} \mathrm{D}_{2}$ & 119780.17 & 126202.87 & 126899.47 & 126651.38 & 126476.24 & 126670.945 \\
\hline $5 \mathrm{~s} 7 \mathrm{p}^{3} \mathrm{P}_{0}^{\mathrm{o}}$ & 116946.39 & 124809.95 & 126608.99 & 126664.90 & 126512.01 & \\
\hline $5 \mathrm{~s} 7 \mathrm{p}^{3} \mathrm{P}_{1}^{\mathrm{o}}$ & 117033.36 & 124882.62 & 126677.44 & 126728.39 & 126575.30 & 126994.866 \\
\hline $5 \mathrm{~s} 7 \mathrm{p}^{3} \mathrm{P}_{2}^{\mathrm{o}}$ & 117247.29 & 125117.43 & 126934.55 & 126983.02 & 126826.69 & 127254.061 \\
\hline $5 \mathrm{~s} 7 \mathrm{p}^{1} \mathrm{P}_{1}^{\mathrm{o}}$ & 118790.12 & 125689.56 & 127366.69 & 127333.16 & 127180.18 & 127573.302 \\
\hline $5 s 8 s^{3} S_{1}$ & 128719.49 & 131265.27 & 132847.63 & 132935.20 & 132776.25 & 133072.509 \\
\hline $5 \mathrm{~s} 8 \mathrm{~s}{ }^{1} \mathrm{~S}_{0}$ & 129180.40 & 131767.57 & 133305.51 & 133405.01 & 133245.69 & 133554.383 \\
\hline $5 \mathrm{~s} 5 \mathrm{f}^{3} \mathrm{~F}_{2}^{\mathrm{o}}$ & 123796.71 & 131866.77 & 133576.51 & 133662.06 & 133499.86 & 133940.984 \\
\hline $5 \mathrm{~s} 5 \mathrm{f}^{3} \mathrm{~F}_{3}^{\mathrm{o}}$ & 123795.69 & 131864.93 & 133581.39 & 133664.78 & 133502.54 & 133944.832 \\
\hline $5 \mathrm{~s} 5 \mathrm{f}^{3} \mathrm{~F}_{4}^{\mathrm{o}}$ & 123794.34 & 131862.75 & 133586.65 & 133672.12 & 133509.81 & 133960.864 \\
\hline $5 \mathrm{~s} 5 \mathrm{f}^{1} \mathrm{~F}_{3}^{\mathrm{o}}$ & 123982.67 & 132012.81 & 133598.24 & 133697.86 & 133535.58 & \\
\hline $5 s 7 d^{3} D_{1}$ & 124477.90 & 133007.73 & 134509.34 & 134590.81 & 134424.80 & 134726.488 \\
\hline $5 \mathrm{~s} 7 \mathrm{~d}^{3} \mathrm{D}_{2}$ & 124494.43 & 133025.14 & 134526.52 & 134607.42 & 134440.93 & 134744.017 \\
\hline $5 s 7 d^{3} D_{3}$ & 124519.45 & 133052.05 & 134553.72 & 134633.93 & 134466.68 & 134771.894 \\
\hline $5 s 7 d^{1} D_{2}$ & 127336.35 & 134218.44 & 135333.37 & 135290.22 & 135120.94 & 135400.325 \\
\hline
\end{tabular}


Table 2. Calculated hyperfine interaction constants for ${ }^{115} \mathrm{In}$ (in MHz) compared with experimental values derived from Fourier transform spectra [1]. In the two last two columns the calculated Landé $g_{J}$ factors are compared with values $g_{L S J}$ derived in pure $L S$ coupling.

\begin{tabular}{|c|c|c|c|c|c|c|}
\hline Level & $A$ & $B$ & $A_{\exp }$ & $B_{\exp }$ & $g_{J}$ & $g_{L S J}$ \\
\hline $\mathrm{s} 5 \mathrm{p}^{3} \mathrm{P}_{1}^{\mathrm{o}}$ & 6832 & -407 & $6949 \pm 24$ & -420 & 1.4982 & 1.5011 \\
\hline $5 \mathrm{~s} 5 \mathrm{p}^{3} \mathrm{P}_{2}^{\mathrm{o}}$ & 5014 & 737 & $5088 \pm 27$ & 629 & 1.5011 & 1.5011 \\
\hline $5 \mathrm{~s} 5 \mathrm{p}^{1} \mathrm{P}_{1}^{\mathrm{o}}$ & -229 & 546 & & & 1.0028 & 1.0000 \\
\hline$s 6 s^{3} S_{1}$ & 11758 & 0.2 & $12049 \pm 12$ & 0 & 2.0021 & 2.0023 \\
\hline $5 \mathrm{~s} 5 \mathrm{~d}^{1} \mathrm{D}_{2}$ & 521 & 611 & $549 \pm 21$ & 570 & 1.0090 & 1.0000 \\
\hline $5 \mathrm{~s} 5 \mathrm{~d}^{3} \mathrm{D}_{1}$ & -4845 & 23.2 & & & 0.5042 & 0.4988 \\
\hline $5 \mathrm{~s} 5 \mathrm{~d}^{3} \mathrm{D}_{2}$ & 1778 & 339 & $1865 \pm 30$ & -600 & 1.1673 & 1.1670 \\
\hline $5 s 5 d^{3} D_{3}$ & 3340 & 94.9 & $3388 \pm 60$ & -60 & 1.3341 & 1.3341 \\
\hline $5 p^{2}{ }^{3} P_{1}$ & -123 & 360 & & & 1.4959 & 1.5011 \\
\hline $5 p^{2}{ }^{3} P_{2}$ & 648 & -776 & $666 \pm 45$ & -629 & 1.4821 & 1.5011 \\
\hline $5 \mathrm{~s} 6 \mathrm{p}^{3} \mathrm{P}_{1}^{\mathrm{o}}$ & 7841 & -74.7 & $8148 \pm 15$ & -480 & 1.4828 & 1.5011 \\
\hline $5 \mathrm{~s} 6 \mathrm{p}^{3} \mathrm{P}_{2}^{\mathrm{o}}$ & 5156 & 155 & $5297 \pm 18$ & 210 & 1.5011 & 1.5011 \\
\hline $5 \mathrm{~s} 6 \mathrm{p}^{1} \mathrm{P}_{1}^{\mathrm{o}}$ & -2245 & 176 & $-2278 \pm 24$ & 149 & 1.0183 & 1.0000 \\
\hline $5 \mathrm{p}^{2}{ }^{1} \mathrm{D}_{2}$ & 455 & 753 & $480 \pm 60$ & 659 & 1.0094 & 1.0000 \\
\hline $5 \mathrm{~s} 7 \mathrm{~s}^{3} \mathrm{~S}_{1}$ & 10927 & -0.006 & $11167 \pm 36$ & -180 & 2.0023 & 2.0023 \\
\hline $5 \mathrm{~s} 4 \mathrm{f}^{3} \mathrm{~F}_{2}^{\mathrm{o}}$ & -3381 & 11.0 & & & 0.6659 & 0.6659 \\
\hline $5 \mathrm{~s} 4 \mathrm{f}^{3} \mathrm{~F}_{3}^{\mathrm{o}}$ & -285 & 12.1 & & & 1.0804 & 1.0835 \\
\hline $5 \mathrm{~s} 4 \mathrm{f}^{3} \mathrm{~F}_{4}^{\mathrm{o}}$ & 2543 & 15.5 & & & 1.2506 & 1.2506 \\
\hline $5 \mathrm{~s} 4 \mathrm{f}^{1} \mathrm{~F}_{3}^{\mathrm{o}}$ & 1141 & 17.2 & $1736 \pm 21$ & -690 & 1.0031 & 1.0000 \\
\hline $5 s 6 d^{3} D_{1}$ & -5094 & 9.4 & $-5228 \pm 24$ & 360 & 0.4988 & 0.4988 \\
\hline $5 s 6 d^{3} D_{2}$ & 1902 & 13.4 & $1931 \pm 24$ & -2008 & 1.1670 & 1.1670 \\
\hline $5 s 6 d^{3} D_{3}$ & 3431 & 25.8 & $3477 \pm 30$ & -1678 & 1.3341 & 1.3341 \\
\hline $5 \mathrm{~s} 6 \mathrm{~d}^{1} \mathrm{D}_{2}$ & -97.3 & 112 & & & 1.0003 & 1.0000 \\
\hline $5 s 7 p^{3} \mathrm{P}_{1}^{\mathrm{o}}$ & 8550 & -24.9 & $9000 \pm 45$ & -1319 & 1.4664 & 1.5011 \\
\hline $5 \mathrm{~s} 7 \mathrm{p}^{3} \mathrm{P}_{2}^{\mathrm{o}}$ & 5156 & 60.0 & $5297 \pm 30$ & 300 & 1.5011 & 1.5011 \\
\hline $5 \mathrm{~s} 7 \mathrm{p}^{1} \mathrm{P}_{1}^{\mathrm{o}}$ & -3209 & 69.2 & $-3424 \pm 27$ & -29 & 1.0348 & 1.0000 \\
\hline $5 s 8 s^{3} S_{1}$ & 10631 & -0.004 & $10894 \pm 30$ & 209 & 2.0023 & 2.0023 \\
\hline $5 s 5 f^{3} F_{2}^{o}$ & -3401 & 6.1 & & & 0.6659 & 0.6659 \\
\hline $5 \mathrm{~s} 5 \mathrm{f}^{3} \mathrm{~F}_{3}^{\mathrm{o}}$ & -399 & 6.5 & & & 1.0798 & 1.0835 \\
\hline $5 \mathrm{~s} 5 \mathrm{f}^{3} \mathrm{~F}_{4}^{\mathrm{o}}$ & 2557 & 8.1 & & & 1.2506 & 1.2506 \\
\hline $5 s 5 f^{1} F_{3}^{o}$ & 1257 & 9.6 & & & 1.0037 & 1.0000 \\
\hline $5 \mathrm{~s} 7 \mathrm{~d}^{3} \mathrm{D}_{1}$ & -5147 & 4.3 & $-5312 \pm 36$ & 869 & 0.4988 & 0.4988 \\
\hline $5 \mathrm{~s} 7 \mathrm{~d}^{3} \mathrm{D}_{2}$ & 1979 & 6.2 & $1970 \pm 36$ & -3837 & 1.1669 & 1.1670 \\
\hline $5 s 7 d^{3} D_{3}$ & 3448 & 11.8 & $3508 \pm 45$ & -2128 & 1.3341 & 1.3341 \\
\hline $5 \mathrm{~s} 7 \mathrm{~d}^{1} \mathrm{D}_{2}$ & -227 & 31.1 & & & 1.0002 & 1.0000 \\
\hline
\end{tabular}

\subsection{The resonance transition}

In table 5 transition energies and weighted oscillator strengths in Coulomb and Babushkin gauges for the $5 \mathrm{~s}^{2}{ }^{1} \mathrm{~S}_{0}-5 \mathrm{~s} 5 \mathrm{p}^{3} \mathrm{P}_{1}^{\mathrm{o}}$ and $5 \mathrm{~s}^{2}{ }^{1} \mathrm{~S}_{0}-5 \mathrm{~s} 5 \mathrm{p}{ }^{1} \mathrm{P}_{1}^{\mathrm{o}}$ transitions are shown. The values were obtained from increasingly large CI calculations including the Breit interaction and leading QED effects. The oscillator strengths converge rapidly with the active sets and basically only two levels of correlation orbitals are needed. Comparing the weighted oscillator strengths with those from the spectrum calculation we see that there is a fairly good consistency. For $5 \mathrm{~s}^{2}{ }^{1} \mathrm{~S}_{0}-5 \mathrm{~s} 5 \mathrm{p}{ }^{3} \mathrm{P}_{1}^{\mathrm{o}}$ there is no difference at all, but for the intercombination transition $5 \mathrm{~s}^{2}{ }^{1} \mathrm{~S}_{0}-$ $5 \mathrm{~s} 5 \mathrm{p}{ }^{3} \mathrm{P}_{1}^{\mathrm{o}}$ the more extensive calculations with correlation orbitals targeted only for the states in the transition, and not for the whole spectrum, give somewhat larger values in better agreement 
Table 3. Calculated weighted oscillator strengths in Coulomb and Babushkin gauges for E1 transitions in In II. The oscillator strengths have been obtained with the theoretical energy differences.

\begin{tabular}{|c|c|c|c|c|}
\hline Transition & $E_{\text {exp }}$ & $\Delta E$ & $g f_{B}$ & $g f_{C}$ \\
\hline $5 \mathrm{~s} 6 \mathrm{~d}^{1} \mathrm{D}_{2}-5 \mathrm{~s} 7 \mathrm{p}^{3} \mathrm{P}_{1}^{\mathrm{o}}$ & 324 & 9 & {$[-3]$} & $1.10[-1]$ \\
\hline $5 \mathrm{~s} 5 \mathrm{f}^{1} \mathrm{~F}_{3}^{\mathrm{o}}-5 \mathrm{~s} 7 \mathrm{~d}^{3} \mathrm{D}_{2}$ & 757 & 905 & $.35[-2]$ & $5.28[-2]$ \\
\hline $5 s 5 f^{1} \mathrm{~F}_{3}^{\mathrm{o}}-5 \mathrm{~s} 7 \mathrm{~d}^{3} \mathrm{D}_{3}$ & 785 & 931 & $.29[-3]$ & $5.35[-3]$ \\
\hline $5 s 5 f^{3} F_{2}^{o}-5 s 7 d^{3} D_{1}$ & 786 & 924 & $1.01[+0]$ & $5.52[-1]$ \\
\hline $5 s 5 f^{3} F_{3}^{o}-5 s 7 d^{3} D_{2}$ & 799 & 938 & $1.42[+0]$ & $7.85[-1]$ \\
\hline $5 s 5 f^{3} \mathrm{~F}_{2}^{\mathrm{o}}-5 \mathrm{~s} 7 \mathrm{~d}^{3} \mathrm{D}_{2}$ & 803 & 941 & $1.89[-1]$ & $1.05[-1]$ \\
\hline $5 s 5 f^{3} F_{4}^{o}-5 s 7 d^{3} D_{3}$ & 811 & 956 & $2.23[+0]$ & $1.26[+0]$ \\
\hline $5 s 5 f^{3} F_{3}^{o}-5 s 7 d^{3} D_{3}$ & 827 & 964 & $1.84[-1]$ & $1.04[-1]$ \\
\hline $5 s 5 f^{3} \mathrm{~F}_{2}^{\mathrm{o}}-5 \mathrm{~s} 7 \mathrm{~d}^{3} \mathrm{D}_{3}$ & 831 & 966 & $5.55[-3]$ & $3.15[-3]$ \\
\hline $5 \mathrm{~s} 6 \mathrm{~d}^{1} \mathrm{D}_{2}-5 \mathrm{~s} 7 \mathrm{p}{ }^{1} \mathrm{P}_{1}^{\mathrm{o}}$ & 902 & 703 & $4.58[-1]$ & $1.19[+0]$ \\
\hline $5 \mathrm{~s} 4 \mathrm{f}^{1} \mathrm{~F}_{3}^{\mathrm{o}}-5 \mathrm{~s} 6 \mathrm{~d}^{3} \mathrm{D}_{2}$ & 1078 & 1264 & $2.70[-2]$ & $4.08[-2]$ \\
\hline $5 \mathrm{~s} 4 \mathrm{f}^{3} \mathrm{~F}_{2}^{\mathrm{o}}-5 \mathrm{~s} 6 \mathrm{~d}^{3} \mathrm{D}_{1}$ & 1100 & 1287 & $3.73[-1]$ & $5.63[-1]$ \\
\hline $5 s 4 f^{3} F_{3}^{o}-5 s 6 d^{3} D_{2}$ & 1129 & 1315 & $5.36[-1]$ & $8.06[-1]$ \\
\hline $5 s 4 f^{1} F_{3}^{o}-5 s 6 d^{3} D_{3}$ & 1131 & 1313 & $2.86[-3]$ & $4.46[-3]$ \\
\hline $5 s 4 f^{3} F_{2}^{o}-5 s 6 d^{3} D_{2}$ & 1134 & 1318 & $7.05[-2]$ & $1.06[-1]$ \\
\hline $5 \mathrm{~s} 4 \mathrm{f}^{3} \mathrm{~F}_{4}^{\mathrm{o}}-5 \mathrm{~s} 6 \mathrm{~d}^{3} \mathrm{D}_{3}$ & 1165 & 1355 & $8.38[-1]$ & $1.25[+0]$ \\
\hline $5 s 4 f^{3} F_{3}^{o}-5 s 6 d^{3} D_{3}$ & 1182 & 1364 & $6.98[-2]$ & $1.04[-1]$ \\
\hline $5 s 4 f^{3} F_{2}^{o}-5 s 6 d^{3} D_{3}$ & 1187 & 1367 & $2.08[-3]$ & $3.11[-3]$ \\
\hline $5 s 5 f^{1} F_{3}^{o}-5 s 7 d^{1} D_{2}$ & 1413 & 1585 & $2.34[+0]$ & $1.72[+0]$ \\
\hline $5 s 5 f^{3} F_{3}^{o}-5 s 7 d^{1} D_{2}$ & 1455 & 1618 & $1.57[-1]$ & $1.15[-1]$ \\
\hline $5 \mathrm{~s} 6 \mathrm{~d}^{3} \mathrm{D}_{1}-5 \mathrm{~s} 7 \mathrm{p}^{3} \mathrm{P}_{0}^{\mathrm{o}}$ & 2189 & 2015 & $4.12[-1]$ & $5.02[-1]$ \\
\hline $5 s 6 d^{3} D_{2}-5 s 7 p^{3} P_{1}^{o}$ & 2218 & 2047 & $8.87[-1]$ & $1.08[+0]$ \\
\hline $5 s 6 d^{3} D_{1}-5 s 7 p^{3} P_{1}^{o}$ & 2252 & 2078 & $2.95[-1]$ & $3.58[-1]$ \\
\hline $5 s 6 d^{3} D_{3}-5 s 7 p^{3} P_{2}^{o}$ & 2424 & 2250 & $1.90[+0]$ & $2.31[+0]$ \\
\hline $5 s 6 d^{3} D_{2}-5 s 7 p^{3} P_{2}^{o}$ & 2477 & 2298 & $3.43[-1]$ & $4.16[-1]$ \\
\hline $5 s 6 d^{3} D_{1}-5 s 7 p^{3} P_{2}^{o}$ & 2511 & 2330 & $2.30[-2]$ & $2.78[-2]$ \\
\hline $5 s 6 d^{3} D_{2}-5 s 7 p^{1} P_{1}^{o}$ & 2797 & 2652 & $6.63[-2]$ & $7.94[-2]$ \\
\hline $5 s 6 d^{3} D_{1}-5 s 7 p^{1} P_{1}^{o}$ & 2 & 3 & {$[-2]$} & $3.17[-2]$ \\
\hline $5 s 4 f^{1} F_{3}^{o}-5 s 6 d^{1} D_{2}$ & 2972 & 3212 & $10[+0]$ & $36[+0]$ \\
\hline $5 \mathrm{~s} 4 \mathrm{f}^{3} \mathrm{~F}_{3}^{\mathrm{o}}-5 \mathrm{~s} 6 \mathrm{~d}^{1} \mathrm{D}_{2}$ & 30 & 4 & $69[-2]$ & $7.12[-2]$ \\
\hline $5 s 7 s^{1} S_{0}-5 s 7 p^{3} P_{1}^{o}$ & 3 & 3199 & {$[-2]$} & $4.56[-2]$ \\
\hline $5 \mathrm{~s} 6 \mathrm{p}^{1} \mathrm{P}_{1}^{\mathrm{o}}-5 \mathrm{p}^{2}{ }^{1} \mathrm{D}_{2}$ & 4105 & 4389 & $5.18[-1]$ & $3.40[-1]$ \\
\hline $5 s 7 s^{1} S_{0}-5 s 7 p^{1} P_{1}^{o}$ & 4200 & 3803 & $5.79[-1]$ & $6.81[-1]$ \\
\hline $5 p^{2}{ }^{3} P_{2}-5 s 6 p^{1} P_{1}^{o}$ & 4215 & 4323 & $2.40[-2]$ & $2.81[-2]$ \\
\hline $5 p^{2}{ }^{3} P_{1}-5 s 6 p^{3} P_{0}^{o}$ & 4414 & 4438 & $1.62[-3]$ & $2.05[-3]$ \\
\hline $5 s 7 s^{3} S_{1}-5 s 7 p^{3} P_{0}^{o}$ & 5489 & 5305 & $5.99[-1]$ & $6.24[-1]$ \\
\hline $5 s 7 p^{1} P_{1}^{o}-5 s 8 s^{3} S_{1}$ & 5499 & 5596 & $1.07[-1]$ & $8.07[-2]$ \\
\hline $5 s 7 s^{3} S_{1}-5 s 7 p^{3} P_{1}^{o}$ & 5552 & 5368 & $1.68[+0]$ & $1.75[+0]$ \\
\hline $5 s 5 d^{3} D_{1}-5 s 6 p^{3} P_{0}^{o}$ & 5574 & 5424 & $2.33[-1]$ & $2.50[-1]$ \\
\hline $5 \mathrm{~s} 5 \mathrm{~d}^{3} \mathrm{D}_{2}-5 \mathrm{~s} 6 \mathrm{p}^{3} \mathrm{P}_{1}^{\mathrm{o}}$ & 5667 & 5518 & $5.20[-1]$ & $5.60[-1]$ \\
\hline $5 p^{2}{ }^{1} S_{0}-5 s 7 p^{3} P_{1}^{o}$ & 5706 & 5210 & $7.33[-2]$ & $7.79[-2]$ \\
\hline $5 \mathrm{~s} 5 \mathrm{~d}^{3} \mathrm{D}_{1}-5 \mathrm{~s} 6 \mathrm{p}^{3} \mathrm{P}_{1}^{\mathrm{o}}$ & 5753 & 5603 & $1.74[-1]$ & $1.87[-1]$ \\
\hline $5 s 7 s^{3} S_{1}-5 s 7 p^{3} P_{2}^{o}$ & 5812 & 5620 & $3.08[+0]$ & $3.21[+0]$ \\
\hline $5 s 7 p^{3} P_{2}^{o}-5 s 8 s^{3} S_{1}$ & 5818 & 5949 & $2.45[+0]$ & $1.82[+0]$ \\
\hline $5 s 7 p^{1} \mathrm{P}_{1}^{\mathrm{o}}-5 \mathrm{~s} 8 \mathrm{~s}{ }^{1} \mathrm{~S}_{0}$ & 5981 & 6065 & $1.21[+0]$ & $1.08[+0]$ \\
\hline $5 \mathrm{~s} 6 \mathrm{p}^{3} \mathrm{P}_{1}^{\mathrm{o}}-5 \mathrm{p}^{2}{ }^{1} \mathrm{D}_{2}$ & 6043 & 6395 & $2.48[-2]$ & $1.88[-2]$ \\
\hline $5 s 7 p^{3} \mathrm{P}_{1}^{\mathrm{o}}-5 \mathrm{~s} 8 \mathrm{~s}^{3} \mathrm{~S}_{1}$ & 6078 & 6200 & $1.30[+0]$ & $9.73[-1]$ \\
\hline $5 \mathrm{~s} 5 \mathrm{~d}^{3} \mathrm{D}_{3}-5 \mathrm{~s} 6 \mathrm{p}^{3} \mathrm{P}_{2}^{\mathrm{o}}$ & 6122 & 5965 & $1.07[+0]$ & $1.15[+0]$ \\
\hline $5 \mathrm{~s} 7 \mathrm{~s}^{3} \mathrm{~S}_{1}-5 \mathrm{~s} 7 \mathrm{p}^{1} \mathrm{P}_{1}^{\mathrm{o}}$ & 6131 & 5973 & $1.31[-1]$ & $1.36[-1]$ \\
\hline
\end{tabular}


Table 3. (Continued.)

\begin{tabular}{|c|c|c|c|c|}
\hline Transition & $\Delta E_{\exp }$ & $\Delta E$ & $g f_{B}$ & $g f_{C}$ \\
\hline $5 \mathrm{~s} 7 \mathrm{p}^{3} \mathrm{P}_{0}^{\mathrm{o}}-5 \mathrm{~s} 8 \mathrm{~s}^{3} \mathrm{~S}_{1}$ & 6141 & 6264 & $4.62[-1]$ & $3.44[-1]$ \\
\hline $5 s 5 d^{3} D_{2}-5 s 6 p^{3} P_{2}^{o}$ & 6255 & 6096 & $1.93[-1]$ & $2.07[-1]$ \\
\hline $5 \mathrm{p}^{21} \mathrm{~S}_{0}-5 \mathrm{~s} 7 \mathrm{p}^{1} \mathrm{P}_{1}^{\mathrm{o}}$ & 6284 & 5815 & $1.03[+0]$ & $1.10[+0]$ \\
\hline $5 s 5 d^{3} D_{1}-5 s 6 p^{3} P_{2}^{o}$ & 6341 & 6182 & $1.26[-2]$ & $1.34[-2]$ \\
\hline $5 \mathrm{~s} 7 \mathrm{p}^{3} \mathrm{P}_{1}^{\mathrm{o}}-5 \mathrm{~s} 8 \mathrm{~s}^{1} \mathrm{~S}_{0}$ & 6560 & 6670 & $7.93[-2]$ & $7.00[-2]$ \\
\hline $5 \mathrm{~s} 7 \mathrm{p}^{1} \mathrm{P}_{1}^{\mathrm{o}}-5 \mathrm{~s} 7 \mathrm{~d}^{3} \mathrm{D}_{1}$ & 7153 & 7244 & $7.39[-2]$ & $6.10[-2]$ \\
\hline $5 \mathrm{~s} 7 \mathrm{p}^{1} \mathrm{P}_{1}^{\mathrm{o}}-5 \mathrm{~s} 7 \mathrm{~d}^{3} \mathrm{D}_{2}$ & 7171 & 7260 & $1.70[-1]$ & $1.38[-1]$ \\
\hline $5 \mathrm{~s} 6 \mathrm{~d}^{1} \mathrm{D}_{2}-5 \mathrm{~s} 5 \mathrm{f}^{3} \mathrm{~F}_{3}^{\mathrm{o}}$ & 7274 & 7026 & $3.47[-1]$ & $3.84[-1]$ \\
\hline $5 s 6 d^{1} D_{2}-5 s 5 f^{1} F_{3}^{o}$ & 7316 & 7059 & $5.59[+0]$ & $6.15[+0]$ \\
\hline $5 \mathrm{~s} 7 \mathrm{p}^{3} \mathrm{P}_{2}^{\mathrm{o}}-5 \mathrm{~s} 7 \mathrm{~d}^{3} \mathrm{D}_{1}$ & 7472 & 7598 & $6.66[-2]$ & $5.46[-2]$ \\
\hline $5 s 7 p^{3} P_{2}^{o}-5 s 7 d^{3} D_{2}$ & 7490 & 7614 & $9.93[-1]$ & $8.14[-1]$ \\
\hline $5 s 7 p^{3} P_{2}^{o}-5 s 7 d^{3} D_{3}$ & 7518 & 7639 & $5.52[+0]$ & $4.53[+0]$ \\
\hline $5 \mathrm{~s} 5 \mathrm{~d}^{3} \mathrm{D}_{2}-5 \mathrm{~s} 6 \mathrm{p}^{1} \mathrm{P}_{1}^{\mathrm{o}}$ & 7605 & 7524 & $2.24[-2]$ & $2.34[-2]$ \\
\hline $5 \mathrm{~s} 5 \mathrm{~d}^{3} \mathrm{D}_{1}-5 \mathrm{~s} 6 \mathrm{p}^{1} \mathrm{P}_{1}^{\mathrm{o}}$ & 7691 & 7609 & $8.19[-3]$ & $8.76[-3]$ \\
\hline $5 s 7 p^{3} P_{1}^{o}-5 s 7 d^{3} D_{1}$ & 7732 & 7849 & $8.82[-1]$ & $7.21[-1]$ \\
\hline $5 \mathrm{~s} 7 \mathrm{p}^{3} \mathrm{P}_{1}^{\mathrm{o}}-5 \mathrm{~s} 7 \mathrm{~d}^{3} \mathrm{D}_{2}$ & 7749 & 7865 & $2.67[+0]$ & $2.19[+0]$ \\
\hline $5 \mathrm{~s} 7 \mathrm{p}^{3} \mathrm{P}_{0}^{\mathrm{o}}-5 \mathrm{~s} 7 \mathrm{~d}^{3} \mathrm{D}_{1}$ & 7795 & 7912 & $1.24[+0]$ & $1.01[+0]$ \\
\hline $5 \mathrm{~s} 7 \mathrm{p}^{1} \mathrm{P}_{1}^{\mathrm{o}}-5 \mathrm{~s} 7 \mathrm{~d}^{1} \mathrm{D}_{2}$ & 7827 & 7940 & $2.96[+0]$ & $2.79[+0]$ \\
\hline $5 s 7 p^{3} P_{1}^{o}-5 s 7 d^{1} D_{2}$ & 8405 & 8545 & $1.50[-1]$ & $1.42[-1]$ \\
\hline $5 s 6 d^{3} D_{3}-5 s 5 f^{3} F_{2}^{o}$ & 9111 & 8923 & $1.70[-2]$ & $1.82[-2]$ \\
\hline $5 \mathrm{~s} 6 \mathrm{~d}^{3} \mathrm{D}_{3}-5 \mathrm{~s} 5 \mathrm{f}^{3} \mathrm{~F}_{3}^{\mathrm{o}}$ & 9115 & 8925 & $5.65[-1]$ & $6.06[-1]$ \\
\hline $5 s 6 d^{3} D_{3}-5 s 5 f^{3} F_{4}^{o}$ & 9131 & 8933 & $6.87[+0]$ & $7.38[+0]$ \\
\hline $5 s 6 d^{3} D_{3}-5 s 5 f^{1} F_{3}^{o}$ & 9157 & 8958 & $2.91[-2]$ & $3.14[-2]$ \\
\hline $5 s 6 d^{3} D_{2}-5 s 5 f^{3} F_{2}^{o}$ & 9164 & 8972 & $5.90[-1]$ & $6.32[-1]$ \\
\hline $5 s 6 d^{3} D_{2}-5 s 5 f^{3} F_{3}^{o}$ & 9168 & 8974 & $4.44[+0]$ & $4.76[+0]$ \\
\hline $5 s 6 d^{3} D_{1}-5 s 5 f^{3} F_{2}^{o}$ & 9198 & 9003 & $3.17[+0]$ & $3.39[+0]$ \\
\hline $5 \mathrm{~s} 6 \mathrm{~d}^{3} \mathrm{D}_{2}-5 \mathrm{~s} 5 \mathrm{f}^{1} \mathrm{~F}_{3}^{\mathrm{o}}$ & 9210 & 9007 & $2.75[-1]$ & $2.95[-1]$ \\
\hline $5 \mathrm{p}^{2}{ }^{1} \mathrm{D}_{2}-5 \mathrm{~s} 4 \mathrm{f}^{3} \mathrm{~F}_{3}^{\mathrm{o}}$ & 9763 & 9420 & $9.14[-2]$ & $9.36[-2]$ \\
\hline $5 \mathrm{p}^{2}{ }^{1} \mathrm{D}_{2}-5 \mathrm{~s} 4 \mathrm{f}^{1} \mathrm{~F}_{3}^{\mathrm{o}}$ & 9814 & 9472 & $2.02[+0]$ & $2.04[+0]$ \\
\hline $5 s 5 d^{1} D_{2}-5 s 6 p^{3} P_{1}^{o}$ & 10214 & 9868 & $2.62[-2]$ & $2.46[-2]$ \\
\hline $5 \mathrm{~s} 6 \mathrm{~s}^{1} \mathrm{~S}_{0}-5 \mathrm{~s} 6 \mathrm{p}^{3} \mathrm{P}_{1}^{\mathrm{o}}$ & 10812 & 10554 & $3.92[-2]$ & $4.28[-2]$ \\
\hline $5 \mathrm{~s} 6 \mathrm{p}^{1} \mathrm{P}_{1}^{\mathrm{o}}-5 \mathrm{p}^{2}{ }^{1} \mathrm{~S}_{0}$ & 11509 & 11963 & $5.14[-1]$ & $4.88[-1]$ \\
\hline $5 s 6{ }^{1} P_{1}^{o}-5 s 7 s^{3} S_{1}$ & 11662 & 11804 & $3.68[-2]$ & $3.20[-2]$ \\
\hline $5 s 4 f^{1} F_{3}^{o}-5 s 7 d^{1} D_{2}$ & 11701 & 11857 & $9.00[-3]$ & $2.58[-2]$ \\
\hline $5 \mathrm{~s} 5 \mathrm{~d}^{1} \mathrm{D}_{2}-5 \mathrm{~s} 6 \mathrm{p}{ }^{1} \mathrm{P}_{1}^{\mathrm{o}}$ & 12152 & 11873 & $7.29[-1]$ & $6.85[-1]$ \\
\hline $5 \mathrm{~s} 6 \mathrm{~s}^{1} \mathrm{~S}_{0}-5 \mathrm{~s} 6 \mathrm{p}^{1} \mathrm{P}_{1}^{\mathrm{o}}$ & 12750 & 12560 & $1.19[+0]$ & $1.28[+0]$ \\
\hline $5 s 6 p^{3} P_{2}^{o}-5 s 7 s^{3} S_{1}$ & 13012 & 13232 & $1.51[+0]$ & $1.36[+0]$ \\
\hline $5 \mathrm{p}^{2}{ }^{1} \mathrm{D}_{2}-5 \mathrm{~s} 7 \mathrm{p}^{3} \mathrm{P}_{1}^{\mathrm{o}}$ & 13110 & 12784 & $2.25[-2]$ & $1.88[-2]$ \\
\hline $5 \mathrm{~s} 6 \mathrm{p}^{3} \mathrm{P}_{1}^{\mathrm{o}}-5 \mathrm{p}^{2}{ }^{1} \mathrm{~S}_{0}$ & 13447 & 13968 & $1.64[-2]$ & $1.63[-2]$ \\
\hline $5 \mathrm{~s} 6 \mathrm{p}{ }^{1} \mathrm{P}_{1}^{\mathrm{o}}-5 \mathrm{~s} 7 \mathrm{~s}{ }^{1} \mathrm{~S}_{0}$ & 13593 & 13974 & $3.35[-1]$ & $3.17[-1]$ \\
\hline $5 s 6 p^{3} P_{1}^{o}-5 s 7 s^{3} S_{1}$ & 13601 & 13810 & $8.29[-1]$ & $7.62[-1]$ \\
\hline $5 \mathrm{p}^{2}{ }^{1} \mathrm{D}_{2}-5 \mathrm{~s} 7 \mathrm{p}^{1} \mathrm{P}_{1}^{\mathrm{o}}$ & 13688 & 13388 & $2.56[-1]$ & $2.22[-1]$ \\
\hline $5 s 6 s^{3} S_{1}-5 s 6 p^{3} P_{0}^{o}$ & 13739 & 13564 & $4.41[-1]$ & $4.61[-1]$ \\
\hline $5 s 6 p^{3} P_{0}^{o}-5 s 7 s^{3} S_{1}$ & 13780 & 13990 & $2.81[-1]$ & $2.60[-1]$ \\
\hline $5 s 6 s^{3} S_{1}-5 s 6 p^{3} P_{1}^{o}$ & 13918 & 13744 & $1.29[+0]$ & $1.34[+0]$ \\
\hline $5 s 6 s^{3} S_{1}-5 s 6 p^{3} P_{2}^{o}$ & 14506 & 14322 & $2.29[+0]$ & $2.39[+0]$ \\
\hline $5 s 6 p^{1} P_{1}^{o}-5 s 6 d^{3} D_{1}$ & 14963 & 15095 & $3.09[-2]$ & $2.92[-2]$ \\
\hline $5 s 6 p^{1} P_{1}^{o}-5 s 6 d^{3} D_{2}$ & 14996 & 15126 & $7.18[-2]$ & $6.76[-2]$ \\
\hline $5 \mathrm{~s} 6 \mathrm{p}^{3} \mathrm{P}_{1}^{\mathrm{o}}-5 \mathrm{~s} 7 \mathrm{~s}^{1} \mathrm{~S}_{0}$ & 15531 & 15980 & $9.99[-3]$ & $1.03[-2]$ \\
\hline $5 s 6 s^{3} S_{1}-5 s 6 p^{1} P_{1}^{o}$ & 15856 & 15750 & $5.24[-2]$ & $5.48[-2]$ \\
\hline $5 s 6 p^{3} P_{2}^{o}-5 s 6 d^{3} D_{1}$ & 16312 & 16522 & $5.16[-2]$ & $4.98[-2]$ \\
\hline
\end{tabular}


Table 3. (Continued.)

\begin{tabular}{|c|c|c|c|c|}
\hline Transition & $\Delta E_{\exp }$ & $\Delta E$ & $g f_{B}$ & $g f_{C}$ \\
\hline $5 \mathrm{~s} 6 \mathrm{p}^{3} \mathrm{P}_{2}^{\mathrm{o}}-5 \mathrm{~s} 6 \mathrm{~d}^{3} \mathrm{D}_{2}$ & 16346 & 16553 & $7.71[-1]$ & $7.44[-1]$ \\
\hline $5 s 6 p^{3} P_{2}^{o}-5 s 6 d^{3} D_{3}$ & 16400 & 16602 & $4.29[+0]$ & $4.14[+0]$ \\
\hline $5 \mathrm{~s} 6 \mathrm{p}^{1} \mathrm{P}_{1}^{\mathrm{o}}-5 \mathrm{~s} 6 \mathrm{~d}^{1} \mathrm{D}_{2}$ & 16891 & 17074 & $2.22[+0]$ & $2.23[+0]$ \\
\hline $5 \mathrm{~s} 6 \mathrm{p}^{3} \mathrm{P}_{1}^{\mathrm{o}}-5 \mathrm{~s} 6 \mathrm{~d}^{3} \mathrm{D}_{1}$ & 16901 & 17100 & $7.15[-1]$ & $6.96[-1]$ \\
\hline $5 s 6 p^{3} P_{1}^{o}-5 s 6 d^{3} D_{2}$ & 16935 & 17132 & $2.15[+0]$ & $2.09[+0]$ \\
\hline $5 \mathrm{~s} 6 \mathrm{p}^{3} \mathrm{P}_{0}^{\mathrm{o}}-5 \mathrm{~s} 6 \mathrm{~d}^{3} \mathrm{D}_{1}$ & 17080 & 17280 & $9.76[-1]$ & $9.53[-1]$ \\
\hline $5 \mathrm{p}^{2}{ }^{3} \mathrm{P}_{2}-5 \mathrm{~s} 4 \mathrm{f}^{3} \mathrm{~F}_{3}^{\mathrm{o}}$ & 18083 & 18133 & $2.12[-2]$ & $2.16[-2]$ \\
\hline $5 \mathrm{p}^{2}{ }^{3} \mathrm{P}_{2}-5 \mathrm{~s} 4 \mathrm{f}^{1} \mathrm{~F}_{3}^{\mathrm{O}}$ & 18134 & 18185 & $1.58[-1]$ & $1.59[-1]$ \\
\hline $5 \mathrm{~s} 6 \mathrm{p}^{3} \mathrm{P}_{1}^{\mathrm{o}}-5 \mathrm{~s} 6 \mathrm{~d}^{1} \mathrm{D}_{2}$ & 18829 & 19080 & $5.53[-2]$ & $5.76[-2]$ \\
\hline $5 \mathrm{p}^{2}{ }^{1} \mathrm{D}_{2}-5 \mathrm{~s} 5 \mathrm{f}^{3} \mathrm{~F}_{3}^{\mathrm{o}}$ & 20060 & 19711 & $4.80[-3]$ & $3.89[-3]$ \\
\hline $5 \mathrm{p}^{2}{ }^{1} \mathrm{D}_{2}-5 \mathrm{~s} 5 \mathrm{f}^{1} \mathrm{~F}_{3}^{\mathrm{o}}$ & 20102 & 19744 & $7.06[-2]$ & $5.91[-2]$ \\
\hline $5 p^{2}{ }^{3} P_{1}-5 s 4 f^{3} F_{2}^{o}$ & 20394 & 20431 & $1.16[-2]$ & $1.17[-2]$ \\
\hline $5 s 5 d^{3} D_{3}-5 s 4 f^{3} F_{2}^{o}$ & 21335 & 21200 & $1.51[-2]$ & $1.49[-2]$ \\
\hline $5 s 5 d^{3} D_{3}-5 s 4 f^{3} F_{3}^{o}$ & 21340 & 21203 & $5.08[-1]$ & $5.04[-1]$ \\
\hline $5 \mathrm{~s} 5 \mathrm{~d}^{3} \mathrm{D}_{3}-5 \mathrm{~s} 4 \mathrm{f}^{3} \mathrm{~F}_{4}^{\mathrm{o}}$ & 21356 & 21212 & $6.17[+0]$ & $6.11[+0]$ \\
\hline $5 \mathrm{~s} 5 \mathrm{~d}^{3} \mathrm{D}_{3}-5 \mathrm{~s} 4 \mathrm{f}^{1} \mathrm{~F}_{3}^{\mathrm{o}}$ & 21391 & 21255 & $2.20[-2]$ & $2.17[-2]$ \\
\hline $5 s 5 d^{3} D_{2}-5 s 4 f^{3} F_{2}^{o}$ & 21468 & 21331 & $5.28[-1]$ & $5.23[-1]$ \\
\hline $5 s 5 d^{3} D_{2}-5 s 4 f^{3} F_{3}^{o}$ & 21473 & 21334 & $4.05[+0]$ & $4.01[+0]$ \\
\hline $5 \mathrm{~s} 5 \mathrm{~d}^{3} \mathrm{D}_{2}-5 \mathrm{~s} 4 \mathrm{f}^{1} \mathrm{~F}_{3}^{\mathrm{o}}$ & 21524 & 21386 & $1.85[-1]$ & $1.84[-1]$ \\
\hline $5 \mathrm{~s} 5 \mathrm{~d}^{3} \mathrm{D}_{1}-5 \mathrm{~s} 4 \mathrm{f}^{3} \mathrm{~F}_{2}^{\mathrm{o}}$ & 21554 & 21416 & $2.84[+0]$ & $2.81[+0]$ \\
\hline $5 s 6 p^{1} P_{1}^{o}-5 s 8 s^{3} S_{1}$ & 23292 & 23374 & $4.00[-3]$ & $2.69[-3]$ \\
\hline $5 s 6{ }^{1} \mathrm{P}_{1}^{\mathrm{o}}-5 \mathrm{~s} 8 \mathrm{~s}{ }^{1} \mathrm{~S}_{0}$ & 23774 & 23844 & $1.05[-1]$ & $1.02[-1]$ \\
\hline $5 s 6 p^{3} P_{2}^{o}-5 s 8 s^{3} S_{1}$ & 24642 & 24802 & $1.97[-1]$ & $1.40[-1]$ \\
\hline $5 \mathrm{~s} 5 \mathrm{~d}^{3} \mathrm{D}_{3}-5 \mathrm{~s} 7 \mathrm{p}^{3} \mathrm{P}_{2}^{\mathrm{o}}$ & 24946 & 24818 & $2.92[-3]$ & $1.02[-3]$ \\
\hline $5 \mathrm{~s} 6 \mathrm{p}^{1} \mathrm{P}_{1}^{\mathrm{o}}-5 \mathrm{~s} 7 \mathrm{~d}^{3} \mathrm{D}_{1}$ & 24946 & 25023 & $3.75[-3]$ & $2.97[-3]$ \\
\hline $5 s 6 p^{1} P_{1}^{o}-5 s 7 d^{3} D_{2}$ & 24964 & 25039 & $7.82[-3]$ & $5.96[-3]$ \\
\hline $5 s 6 p^{3} P_{1}^{o}-5 s 8 s^{3} S_{1}$ & 25231 & 25380 & $1.14[-1]$ & $8.41[-2]$ \\
\hline $5 \mathrm{~s} 6 \mathrm{p}^{3} \mathrm{P}_{0}^{\mathrm{o}}-5 \mathrm{~s} 8 \mathrm{~s}^{3} \mathrm{~S}_{1}$ & 25410 & 25560 & $3.95[-2]$ & $2.93[-2]$ \\
\hline $5 s 6 p^{1} P_{1}^{o}-5 s 7 d^{1} D_{2}$ & 25620 & 25719 & $4.59[-1]$ & $4.55[-1]$ \\
\hline $5 \mathrm{~s} 6 \mathrm{p}^{3} \mathrm{P}_{1}^{\mathrm{o}}-5 \mathrm{~s} 8 \mathrm{~s}^{1} \mathrm{~S}_{0}$ & 25712 & 25849 & $4.09[-3]$ & $4.24[-3]$ \\
\hline $5 \mathrm{~s} 5 \mathrm{~d}^{1} \mathrm{D}_{2}-5 \mathrm{~s} 4 \mathrm{f}^{3} \mathrm{~F}_{3}^{\mathrm{o}}$ & 26020 & 25684 & $1.20[-1]$ & $1.17[-1]$ \\
\hline $5 \mathrm{~s} 5 \mathrm{~d}^{1} \mathrm{D}_{2}-5 \mathrm{~s} 4 \mathrm{f}^{1} \mathrm{~F}_{3}^{\mathrm{o}}$ & 26071 & 25736 & $2.87[+0]$ & $2.81[+0]$ \\
\hline $5 \mathrm{~s} 6 \mathrm{p}^{3} \mathrm{P}_{2}^{\mathrm{o}}-5 \mathrm{~s} 7 \mathrm{~d}^{3} \mathrm{D}_{1}$ & 26296 & 26450 & $7.55[-3]$ & $6.20[-3]$ \\
\hline $5 \mathrm{~s} 6 \mathrm{p}^{3} \mathrm{P}_{2}^{\mathrm{o}}-5 \mathrm{~s} 7 \mathrm{~d}^{3} \mathrm{D}_{2}$ & 26314 & 26467 & $1.13[-1]$ & $9.34[-2]$ \\
\hline $5 s 6 p^{3} P_{2}^{o}-5 s 7 d^{3} D_{3}$ & 26342 & 26492 & $6.42[-1]$ & $5.28[-1]$ \\
\hline $5 \mathrm{~s} 6 \mathrm{p}^{3} \mathrm{P}_{1}^{\mathrm{o}}-5 \mathrm{~s} 7 \mathrm{~d}^{3} \mathrm{D}_{1}$ & 26884 & 27029 & $1.11[-1]$ & $9.31[-2]$ \\
\hline $5 s 6 p^{3} P_{1}^{o}-5 s 7 d^{3} D_{2}$ & 26902 & 27045 & $3.39[-1]$ & $2.84[-1]$ \\
\hline $5 \mathrm{~s} 6 \mathrm{p}^{3} \mathrm{P}_{0}^{\mathrm{o}}-5 \mathrm{~s} 7 \mathrm{~d}^{3} \mathrm{D}_{1}$ & 27064 & 27208 & $1.54[-1]$ & $1.30[-1]$ \\
\hline $5 \mathrm{~s} 6 \mathrm{p}^{3} \mathrm{P}_{1}^{\mathrm{o}}-5 \mathrm{~s} 7 \mathrm{~d}^{1} \mathrm{D}_{2}$ & 27558 & 27725 & $1.33[-2]$ & $1.40[-2]$ \\
\hline $5 \mathrm{p}^{2}{ }^{3} \mathrm{P}_{2}-5 \mathrm{~s} 5 \mathrm{f}^{1} \mathrm{~F}_{3}^{\mathrm{o}}$ & 28422 & 28457 & $7.45[-3]$ & $8.17[-3]$ \\
\hline $5 s 5 d^{1} D_{2}-5 s 7 p^{3} P_{1}^{o}$ & 29366 & 29047 & $3.06[-3]$ & $2.36[-3]$ \\
\hline $5 s 5 d^{1} D_{2}-5 s 7 p^{1} P_{1}^{o}$ & 29945 & 29652 & $5.80[-2]$ & $4.67[-2]$ \\
\hline $5 s 5 p^{1} P_{1}^{o}-5 s 6 s^{3} S_{1}$ & 30885 & 30871 & $4.08[-3]$ & $4.04[-3]$ \\
\hline $5 s 5 d^{3} D_{3}-5 s 5 f^{3} F_{2}^{o}$ & 31633 & 31491 & $1.29[-3]$ & $1.35[-3]$ \\
\hline $5 \mathrm{~s} 5 \mathrm{~d}^{3} \mathrm{D}_{3}-5 \mathrm{~s} 5 \mathrm{f}^{3} \mathrm{~F}_{3}^{\mathrm{o}}$ & 31636 & 31494 & $4.38[-2]$ & $4.57[-2]$ \\
\hline $5 \mathrm{~s} 5 \mathrm{~d}^{3} \mathrm{D}_{3}-5 \mathrm{~s} 5 \mathrm{f}^{3} \mathrm{~F}_{4}^{\mathrm{o}}$ & 31652 & 31501 & $5.57[-1]$ & $5.79[-1]$ \\
\hline $5 s 5 d^{3} D_{3}-5 s 5 f^{1} F_{3}^{o}$ & 31678 & 31527 & $2.66[-3]$ & $2.73[-3]$ \\
\hline $5 \mathrm{~s} 5 \mathrm{~d}^{3} \mathrm{D}_{2}-5 \mathrm{~s} 5 \mathrm{f}^{3} \mathrm{~F}_{2}^{\mathrm{o}}$ & 31766 & 31622 & $4.68[-2]$ & $4.87[-2]$ \\
\hline $5 \mathrm{~s} 5 \mathrm{~d}^{3} \mathrm{D}_{2}-5 \mathrm{~s} 5 \mathrm{f}^{3} \mathrm{~F}_{3}^{\mathrm{o}}$ & 31770 & 31625 & $3.63[-1]$ & $3.78[-1]$ \\
\hline $5 s 5 d^{3} D_{2}-5 s 5 f^{1} F_{3}^{o}$ & 31812 & 31658 & $2.11[-2]$ & $2.20[-2]$ \\
\hline $5 s 5 d^{3} D_{1}-5 s 5 f^{3} F_{2}^{o}$ & 31852 & 31708 & $2.57[-1]$ & $2.68[-1]$ \\
\hline
\end{tabular}


Table 3. (Continued.)

\begin{tabular}{|c|c|c|c|c|}
\hline Transition & $\Delta E_{\exp }$ & $\Delta E$ & $g f_{B}$ & $g f_{C}$ \\
\hline $5 s 6 s^{3} S_{1}-5 s 7 p^{3} P_{1}^{o}$ & 071 & 32924 & $3.23[-3]$ & $2.05[-3]$ \\
\hline $5 s 6 s^{3} S_{1}-5 s 7 p^{3} P_{2}^{o}$ & 33330 & 33175 & $1.39[-2]$ & $1.01[-2]$ \\
\hline $5 s 6 s^{3} S_{1}-5 s 7 p^{1} P_{1}^{o}$ & 33649 & 33528 & $1.07[-3]$ & $8.56[-4]$ \\
\hline $5 \mathrm{~s} 5 \mathrm{p}{ }^{1} \mathrm{P}_{1}^{\mathrm{o}}-5 \mathrm{~s} 6 \mathrm{~s}^{1} \mathrm{~S}_{0}$ & 33992 & 34061 & $4.59[-1]$ & $4.50[-1]$ \\
\hline $5 s 5 p^{1} P_{1}^{o}-5 s 5 d^{1} D_{2}$ & 34590 & 34747 & $1.14[-1]$ & $8.69[-2]$ \\
\hline $5 s 5 d^{1} D_{2}-5 s 5 f^{3} F_{3}^{o}$ & 36316 & 35975 & $2.42[-2]$ & $2.37[-2]$ \\
\hline$s 5 d^{1} D_{2}-5 s 5 f{ }^{1} F_{3}^{o}$ & 36358 & 36008 & $4.67[-1]$ & $4.65[-1]$ \\
\hline $5 \mathrm{~s} 5 \mathrm{p}^{1} \mathrm{P}_{1}^{\mathrm{o}}-5 \mathrm{p}^{2}{ }^{3} \mathrm{P}_{0}$ & 38569 & 38402 & $1.42[-3]$ & $1.05[-3]$ \\
\hline $5 s 5 p^{1} P_{1}^{o}-5 s 5 d^{3} D_{1}$ & 39050 & 39012 & $3.56[-3]$ & $3.61[-3]$ \\
\hline $5 \mathrm{~s} 5 \mathrm{p}^{1} \mathrm{P}_{1}^{\mathrm{o}}-5 \mathrm{~s} 5 \mathrm{~d}^{3} \mathrm{D}_{2}$ & 39136 & 39097 & $7.56[-3]$ & $7.40[-3]$ \\
\hline $5 \mathrm{~s} 5 \mathrm{p}^{1} \mathrm{P}_{1}^{\mathrm{o}}-5 \mathrm{p}^{2}{ }^{3} \mathrm{P}_{1}$ & 40210 & 39997 & $1.37[-3]$ & $1.47[-3]$ \\
\hline $5 s 5 p^{1} P_{1}^{o}-5 p^{2}{ }^{3} P_{2}$ & 42527 & 42298 & $6.98[-2]$ & $6.97[-2]$ \\
\hline $5 s^{2}{ }^{1} S_{0}-5 s 5 p^{3} P_{1}^{o}$ & 43351 & 43141 & $4.91[-3]$ & $5.86[-3]$ \\
\hline $5 s 5 p^{3} P_{2}^{o}-5 s 6 s^{3} S_{1}$ & 48095 & 48076 & $8.08[-1]$ & $7.76[-1]$ \\
\hline $5 \mathrm{~s} 5 \mathrm{p}^{3} \mathrm{P}_{1}^{\mathrm{o}}-5 \mathrm{~s} 6 \mathrm{~s}^{3} \mathrm{~S}_{1}$ & 50573 & 50509 & $4.80[-1]$ & $4.62[-1]$ \\
\hline $5 \mathrm{~s} 5 \mathrm{p}^{1} \mathrm{P}_{1}^{\mathrm{o}}-5 \mathrm{p}^{2}{ }^{1} \mathrm{D}_{2}$ & 50846 & 51011 & $3.68[+0]$ & $3.87[+0]$ \\
\hline $5 \mathrm{~s} 5 \mathrm{p}^{3} \mathrm{P}_{0}^{\mathrm{o}}-5 \mathrm{~s} 6 \mathrm{~s}^{3} \mathrm{~S}_{1}$ & 51648 & 51566 & $1.61[-1]$ & $1.56[-1]$ \\
\hline $5 \mathrm{~s} 5 \mathrm{p}^{3} \mathrm{P}_{2}^{\mathrm{o}}-5 \mathrm{~s} 5 \mathrm{~d}^{1} \mathrm{D}_{2}$ & 51799 & 51952 & $3.00[-2]$ & $3.25[-2]$ \\
\hline $5 \mathrm{~s} 5 \mathrm{p}^{3} \mathrm{P}_{1}^{\mathrm{o}}-5 \mathrm{~s} 6 \mathrm{~s}^{1} \mathrm{~S}_{0}$ & 53680 & 53699 & $2.45[-3]$ & $2.55[-3]$ \\
\hline $5 \mathrm{~s} 5 \mathrm{p}^{3} \mathrm{P}_{1}^{\mathrm{o}}-5 \mathrm{~s} 5 \mathrm{~d}^{1} \mathrm{D}_{2}$ & 54278 & 54385 & $9.31[-3]$ & $1.07[-2]$ \\
\hline $5 \mathrm{~s} 5 \mathrm{p}^{3} \mathrm{P}_{2}^{\mathrm{o}}-5 \mathrm{~s} 5 \mathrm{~d}^{3} \mathrm{D}_{1}$ & 56260 & 56216 & $2.26[-2]$ & $2.23[-2]$ \\
\hline $5 s 5 p^{3} \mathrm{P}_{2}^{\mathrm{o}}-5 \mathrm{~s} 5 \mathrm{~d}^{3} \mathrm{D}_{2}$ & 56346 & 56301 & $5.51[-1]$ & $5.54[-1]$ \\
\hline $5 s 5 p^{3} P_{2}^{o}-5 s 5 d^{3} D_{3}$ & 56479 & 56432 & $3.43[+0]$ & $3.47[+0]$ \\
\hline $5 \mathrm{~s} 5 \mathrm{p}^{3} \mathrm{P}_{2}^{\mathrm{o}}-5 \mathrm{p}^{2}{ }^{3} \mathrm{P}_{1}$ & 57420 & 57202 & $6.60[-1]$ & $7.09[-1]$ \\
\hline $5 s 5 p^{1} P_{1}^{o}-5 p^{2}{ }^{1} S_{0}$ & 58251 & 58584 & $9.98[-2]$ & $9.66[-2]$ \\
\hline $5 s 5 p^{3} P_{1}^{0}-5 p^{2}{ }^{3} P_{0}$ & 58257 & 39 & $5.04[-1]$ & $5.41[-1]$ \\
\hline $5 s 5 p^{3} P_{1}^{o}-5 s 5 d^{3} D_{1}$ & 58 & 50 & $5.37[-1]$ & $5.40[-1]$ \\
\hline $5 \mathrm{~s} 5 \mathrm{p}^{3} \mathrm{P}_{1}^{\mathrm{o}}-5 \mathrm{~s} 5 \mathrm{~d}^{3} \mathrm{D}_{2}$ & 58824 & 58735 & $1.86[+0]$ & $1.88[+0]$ \\
\hline $5 \mathrm{~s} 5 \mathrm{p}^{3} \mathrm{P}_{2}^{\mathrm{o}}-5 \mathrm{p}^{2}{ }^{3} \mathrm{P}_{2}$ & 59 & 502 & $1.94[+0]$ & $2.08[+0]$ \\
\hline $5 \mathrm{~s} 5 \mathrm{p}^{3} \mathrm{P}_{0}^{\mathrm{o}}-5 \mathrm{~s} 5 \mathrm{~d}^{3} \mathrm{D}_{1}$ & 598 & 59706 & $8.77[-1]$ & $8.89[-1]$ \\
\hline $5 s 5 p^{3} P_{1}^{o}-5 p^{2}{ }^{3} P_{1}$ & 598 & 59635 & $4.39[-1]$ & $4.71[-1]$ \\
\hline $5 \mathrm{~s} 5 \mathrm{p}^{1} \mathrm{P}_{1}^{\mathrm{o}}-5 \mathrm{~s} 7 \mathrm{~s}^{1} \mathrm{~S}_{0}$ & 6 & 96 & $5.62[-1]$ & $5.49[-1]$ \\
\hline $5 s 5 p^{3} \mathrm{P}_{0}^{\mathrm{o}}-5 \mathrm{p}^{2}{ }^{3} \mathrm{P}_{1}$ & 60973 & 60692 & $4.40[-1]$ & $4.77[-1]$ \\
\hline $5 \mathrm{~s} 5 \mathrm{p}^{3} \mathrm{P}_{1}^{\mathrm{o}}-5 \mathrm{p}^{2}{ }^{3} \mathrm{P}_{2}$ & 62215 & 61936 & $6.15[-1]$ & $6.63[-1]$ \\
\hline $5 \mathrm{~s}^{2}{ }^{1} \mathrm{~S}_{0}-5 \mathrm{~s} 5 \mathrm{p}{ }^{1} \mathrm{P}_{1}^{\mathrm{o}}$ & 63039 & 62779 & $1.56[+0]$ & $1.70[+0]$ \\
\hline $5 s 5 p^{1} P_{1}^{o}-5 s 6 d^{1} D_{2}$ & 63632 & 63696 & $9.62[-1]$ & $1.00[+0]$ \\
\hline $5 \mathrm{~s} 5 \mathrm{p}^{3} \mathrm{P}_{2}^{\mathrm{o}}-5 \mathrm{p}^{2}{ }^{1} \mathrm{D}_{2}$ & 68056 & 68216 & $4.05[-2]$ & $4.34[-2]$ \\
\hline $5 s 5 p^{1} P_{1}^{o}-5 s 8 s^{1} S_{0}$ & 70516 & 70466 & $3.57[-2]$ & $3.77[-2]$ \\
\hline $5 s 5 p^{1} P_{1}^{o}-5 s 7 d^{1} D_{2}$ & 72362 & 72341 & $2.59[-1]$ & $2.76[-1]$ \\
\hline $5 \mathrm{~s} 5 \mathrm{p}^{3} \mathrm{P}_{2}^{\mathrm{o}}-5 \mathrm{~s} 7 \mathrm{~s}^{3} \mathrm{~S}_{1}$ & 75613 & 75631 & $1.17[-1]$ & $1.05[-1]$ \\
\hline $5 \mathrm{~s} 5 \mathrm{p}^{3} \mathrm{P}_{1}^{\mathrm{o}}-5 \mathrm{p}^{2}{ }^{1} \mathrm{~S}_{0}$ & 77939 & 78222 & $1.98[-3]$ & $2.28[-3]$ \\
\hline $5 \mathrm{~s} 5 \mathrm{p}^{3} \mathrm{P}_{1}^{\mathrm{o}}-5 \mathrm{~s} 7 \mathrm{~s}^{3} \mathrm{~S}_{1}$ & 78092 & 78064 & $6.57[-2]$ & $5.86[-2]$ \\
\hline $5 s 5 p^{3} P_{2}^{o}-5 s 6 d^{3} D_{1}$ & 78913 & 78921 & $4.17[-3]$ & $4.37[-3]$ \\
\hline $5 \mathrm{~s} 5 \mathrm{p}^{3} \mathrm{P}_{2}^{\mathrm{o}}-5 \mathrm{~s} 6 \mathrm{~d}^{3} \mathrm{D}_{2}$ & 78947 & 78952 & $6.29[-2]$ & $6.57[-2]$ \\
\hline $5 s 5 p^{3} P_{2}^{o}-5 s 6 d^{3} D_{3}$ & 79001 & 79001 & $3.49[-1]$ & $3.63[-1]$ \\
\hline $5 \mathrm{~s} 5 \mathrm{p}^{3} \mathrm{P}_{0}^{\mathrm{o}}-5 \mathrm{~s} 7 \mathrm{~s}^{3} \mathrm{~S}_{1}$ & 79167 & 79121 & $2.12[-2]$ & $1.89[-2]$ \\
\hline $5 \mathrm{~s} 5 \mathrm{p}^{3} \mathrm{P}_{1}^{\mathrm{o}}-5 \mathrm{~s} 6 \mathrm{~d}^{3} \mathrm{D}_{1}$ & 81392 & 81354 & $6.37[-2]$ & $6.65[-2]$ \\
\hline $5 s 5 p^{3} P_{1}^{o}-5 s 6 d^{3} D_{2}$ & 81426 & 81385 & $1.91[-1]$ & $1.99[-1]$ \\
\hline $5 \mathrm{~s} 5 \mathrm{p}^{3} \mathrm{P}_{0}^{\mathrm{o}}-5 \mathrm{~s} 6 \mathrm{~d}^{3} \mathrm{D}_{1}$ & 82467 & 82411 & $8.60[-2]$ & $8.96[-2]$ \\
\hline $5 \mathrm{~s} 5 \mathrm{p}^{3} \mathrm{P}_{1}^{\mathrm{o}}-5 \mathrm{~s} 6 \mathrm{~d}^{1} \mathrm{D}_{2}$ & 83320 & 83334 & $2.46[-3]$ & $2.61[-3]$ \\
\hline $5 s 5 p^{3} P_{2}^{o}-5 s 8 s^{3} S_{1}$ & 87243 & 87201 & $3.79[-2]$ & $3.47[-2]$ \\
\hline
\end{tabular}


Table 3. (Continued.)

\begin{tabular}{lllll}
\hline Transition & $\Delta E_{\text {exp }}$ & $\Delta E$ & $g f_{B}$ & $g f_{C}$ \\
\hline $5 \mathrm{~s} 5 \mathrm{p}^{3} \mathrm{P}_{2}^{\mathrm{o}}-5 \mathrm{~s} 7 \mathrm{~d}^{3} \mathrm{D}_{1}$ & 88897 & 88849 & $1.13[-3]$ & $1.22[-3]$ \\
$5 \mathrm{~s} 5 \mathrm{p}{ }^{3} \mathrm{P}_{2}^{\mathrm{o}}-5 \mathrm{~s} 7 \mathrm{~d}^{3} \mathrm{D}_{2}$ & 88915 & 88865 & $1.72[-2]$ & $1.85[-2]$ \\
$5 \mathrm{~s} 5 \mathrm{p}^{3} \mathrm{P}_{2}^{\mathrm{o}}-5 \mathrm{~s} 7 \mathrm{~d}^{3} \mathrm{D}_{3}$ & 88943 & 88891 & $9.69[-2]$ & $1.04[-1]$ \\
$5 \mathrm{~s} 5 \mathrm{p}{ }^{3} \mathrm{P}_{1}^{\mathrm{O}}-5 \mathrm{~s} 8 \mathrm{~s}^{3} \mathrm{~S}_{1}$ & 89722 & 89634 & $2.14[-2]$ & $1.98[-2]$ \\
$5 \mathrm{~s} 5 \mathrm{p}^{3} \mathrm{P}_{0}^{\mathrm{o}}-5 \mathrm{~s} 8 \mathrm{~s}^{3} \mathrm{~S}_{1}$ & 90797 & 90691 & $6.98[-3]$ & $6.47[-3]$ \\
$5 \mathrm{~s} 5 \mathrm{p}^{3} \mathrm{P}_{1}^{\mathrm{o}}-5 \mathrm{~s} 7 \mathrm{~d}^{3} \mathrm{D}_{1}$ & 91376 & 91282 & $1.78[-2]$ & $1.93[-2]$ \\
$5 \mathrm{~s} 5 \mathrm{p}^{3} \mathrm{P}_{1}^{\mathrm{o}}-5 \mathrm{~s} 7 \mathrm{~d}^{3} \mathrm{D}_{2}$ & 91393 & 91299 & $5.41[-2]$ & $5.86[-2]$ \\
$5 \mathrm{~s} 5 \mathrm{p}{ }^{3} \mathrm{P}_{0}^{\mathrm{o}}-5 \mathrm{~s} 7 \mathrm{~d}^{3} \mathrm{D}_{1}$ & 92450 & 92339 & $2.43[-2]$ & $2.64[-2]$ \\
\hline
\end{tabular}

with experiment. The good overall agreement, however, strengthens our confidence in the spectrum calculations, indicating that these include the same correlation effects as do the calculations targeted for the individual states. In table 5 values from the HFR calculations of Biémont and Zeippen [15] and the HFR and MCDHF calculations of Biémont et al [16] are also presented. The MCDHF calculations include the same correlation effects as the present calculations but the ${ }^{1} \mathrm{P}_{1}^{\mathrm{o}}$ state is separately optimized in one calculation and the three ${ }^{3} \mathrm{P}^{\mathrm{o}}$ states in another. One notes the large effect of the optimization scheme on the oscillator strength of the allowed transition in Coulomb gauge. The updated HFR calculation for the intercombination transition is in very good agreement with the present value from the CI calculation.

Hyperfine structure constants obtained from the CI wavefunctions including spinpolarization are displayed in table 6. Both diagonal and off-diagonal parameters are shown. The diagonal and off-diagonal constants show a similar convergence pattern and are fairly stable after three layers of correlation orbitals. The hyperfine constants from the more extensive calculations with correlation orbitals targeted for the particular state seem to be somewhat more accurate than the constants from the spectrum calculation. The differences between the two sets of calculations are however small.

In table 7 the differences $\Delta S_{\mathrm{nms}}, \Delta S_{\mathrm{sms}}$ and $\Delta \rho(0)$ between the upper and lower state isotope shift parameters in the $5 \mathrm{~s}^{2}{ }^{1} \mathrm{~S}_{0}-5 \mathrm{~s} 5 \mathrm{p}^{3} \mathrm{P}_{1}^{\mathrm{o}}$ transition are shown as functions of the increasing active set of orbitals. The computed quantities converge after three layers of correlation orbitals. Combining the computed differences in electronic quantities with differences in nuclear quantities according to equation (9) we obtain the isotope shift $\Delta v=v_{115}-v_{113}$. The difference in the root-mean-square radius between ${ }^{115} \mathrm{In}$ and ${ }^{113} \mathrm{In}$ is $-0.128(8) \mathrm{fm}^{2}$ and was taken from Eberz et al [32]. The calculated isotope shift is compared with the experimental shift in table 8 . As suggested by Peik et al, the shift is dominated by the volume effect. Accounting for the uncertainties in the difference in the root-mean-square radius the calculated total shift $-0.515 \mathrm{GHz}$ is within the error bars of the experiment. The main uncertainty in the calculation comes from $\Delta S_{\mathrm{sms}}$. This quantity is sensitive to correlation effects in the core and is known to be extremely difficult to compute accurately.

\subsection{The $5 \mathrm{~s}^{2}{ }^{1} \mathrm{~S}_{0}-5 \mathrm{~s} 5 \mathrm{p}^{3} \mathrm{P}_{0}^{\mathrm{o}}$ hyperfine induced transition}

The hyperfine interaction, although weak, not only shifts and splits individual $J$ levels, but also mixes wavefunctions with different $J$ quantum numbers. The wavefunction for the $5 \mathrm{~s} 5 \mathrm{p}^{3} \mathrm{P}_{0}^{\mathrm{o}}$ state can, when the hyperfine interaction is included, be written as

$$
\left|{ }^{\prime} 5 \mathrm{~s} 5 \mathrm{p}^{3} \mathrm{P}_{0}^{\mathrm{o}} I F '\right\rangle=c_{0}\left|5 \mathrm{~s} 5 \mathrm{p}^{3} \mathrm{P}_{0}^{\mathrm{o}} I F\right\rangle+\sum_{i} c_{i}\left|\gamma_{i} J_{i} I F\right\rangle,
$$


Table 4. Comparison of the present calculated weighted oscillator strengths in Coulomb and Babushkin gauges with previous theoretical and experimental results.

\begin{tabular}{|c|c|c|c|c|c|}
\hline Transition & $\Delta E_{\exp }$ & $g f_{B}$ & $g f_{C}$ & $g f^{\mathrm{a}}$ & \\
\hline $5 s^{2}{ }^{1} S_{0}-5 s 5 p^{3} P_{1}^{o}$ & 43351 & $4.91[-3]$ & $5.86[-3]$ & $7.3[-3]$ & $5.44(45)[-3]^{\mathrm{b}}$ \\
\hline $5 s^{2}{ }^{1} S_{0}-5 s 5 p^{1} P_{1}^{o}$ & 63039 & $1.56[+0]$ & $1.70[+0]$ & $1.64[+0]$ & $1.43(9)[+0]^{\mathrm{c}}$ \\
\hline $5 s^{2}{ }^{1} S_{0}-5 s 6 p^{1} P_{1}^{o}$ & 109780 & $7.80[-4]$ & $3.79[-3]$ & $2.7[-3]$ & \\
\hline $5 \mathrm{~s} 5 \mathrm{p}{ }^{1} \mathrm{P}_{1}^{\mathrm{o}}-5 \mathrm{~s} 6 \mathrm{~s}{ }^{1} \mathrm{~S}_{0}$ & 33992 & $4.59[-1]$ & $4.50[-1]$ & $6.81[-1]$ & \\
\hline $5 \mathrm{~s} 6 \mathrm{~s}^{1} \mathrm{~S}_{0}-5 \mathrm{~s} 6 \mathrm{p}{ }^{1} \mathrm{P}_{1}^{\mathrm{o}}$ & 12750 & $1.19[+0]$ & $1.28[+0]$ & $1.32[+0]$ & \\
\hline $5 s 5 p^{3} P_{2}^{o}-5 s 6 s^{3} S_{1}$ & 48095 & $8.08[-1]$ & $7.76[-1]$ & $8.15[-1]$ & \\
\hline $5 \mathrm{~s} 5 \mathrm{p}^{3} \mathrm{P}_{1}^{\mathrm{o}}-5 \mathrm{~s} 6 \mathrm{~s}^{3} \mathrm{~S}_{1}$ & 50573 & $4.80[-1]$ & $4.62[-1]$ & $5.13[-1]$ & \\
\hline $5 \mathrm{~s} 5 \mathrm{p}^{3} \mathrm{P}_{0}^{\mathrm{o}}-5 \mathrm{~s} 6 \mathrm{~s}^{3} \mathrm{~S}_{1}$ & 51648 & $1.61[-1]$ & $1.56[-1]$ & $1.76[-1]$ & \\
\hline $5 \mathrm{~s} 5 \mathrm{p}^{3} \mathrm{P}_{2}^{\mathrm{o}}-5 \mathrm{~s} 7 \mathrm{~s}^{3} \mathrm{~S}_{1}$ & 75613 & $1.17[-1]$ & $1.05[-1]$ & $1.2[-1]$ & \\
\hline $5 \mathrm{~s} 5 \mathrm{p}^{3} \mathrm{P}_{1}^{\mathrm{o}}-5 \mathrm{~s} 7 \mathrm{~s}^{3} \mathrm{~S}_{1}$ & 78092 & $6.57[-2]$ & $5.86[-2]$ & $7.5[-2]$ & \\
\hline $5 \mathrm{~s} 5 \mathrm{p}^{3} \mathrm{P}_{0}^{\mathrm{o}}-5 \mathrm{~s} 7 \mathrm{~s}^{3} \mathrm{~S}_{1}$ & 79167 & $2.12[-2]$ & $1.89[-2]$ & $2.5[-2]$ & \\
\hline $5 \mathrm{~s} 5 \mathrm{p}^{3} \mathrm{P}_{2}^{\mathrm{o}}-5 \mathrm{~s} 8 \mathrm{~s}^{3} \mathrm{~S}_{1}$ & 87243 & $3.79[-2]$ & $3.47[-2]$ & $4.5[-2]$ & \\
\hline $5 \mathrm{~s} 5 \mathrm{p}^{3} \mathrm{P}_{1}^{\mathrm{o}}-5 \mathrm{~s} 8 \mathrm{~s}^{3} \mathrm{~S}_{1}$ & 89722 & $2.14[-2]$ & $1.98[-2]$ & $2.8[-2]$ & \\
\hline $5 \mathrm{~s} 5 \mathrm{p}^{3} \mathrm{P}_{0}^{\mathrm{o}}-5 \mathrm{~s} 8 \mathrm{~s}^{3} \mathrm{~S}_{1}$ & 90797 & $6.98[-3]$ & $6.47[-3]$ & $9.5[-3]$ & \\
\hline $5 s 6 s^{3} S_{1}-5 s 6 p^{3} P_{2}^{o}$ & 14506 & $2.29[+0]$ & $2.39[+0]$ & $2.46[+0]$ & \\
\hline $5 s^{2} s^{3} S_{1}-5 s 6 p^{3} P_{1}^{o}$ & 13918 & $1.29[+0]$ & $1.34[+0]$ & $1.37[+0]$ & \\
\hline $5 \mathrm{~s} 6 \mathrm{~s}^{3} \mathrm{~S}_{1}-5 \mathrm{~s} 6 \mathrm{p}^{3} \mathrm{P}_{0}^{\mathrm{o}}$ & 13739 & $4.41[-1]$ & $4.61[-1]$ & $4.65[-1]$ & \\
\hline $5 \mathrm{~s} 5 \mathrm{p}^{3} \mathrm{P}_{2}^{\mathrm{o}}-5 \mathrm{~s} 5 \mathrm{~d}^{3} \mathrm{D}_{3}$ & 56479 & $3.43[+0]$ & $3.47[+0]$ & $3.36[+0]$ & \\
\hline $5 \mathrm{~s} 5 \mathrm{p}^{3} \mathrm{P}_{2}^{\mathrm{o}}-5 \mathrm{~s} 5 \mathrm{~d}^{3} \mathrm{D}_{2}$ & 56346 & $5.51[-1]$ & $5.54[-1]$ & $5.90[-1]$ & \\
\hline $5 \mathrm{~s} 5 \mathrm{p}^{3} \mathrm{P}_{2}^{\mathrm{o}}-5 \mathrm{~s} 5 \mathrm{~d}^{3} \mathrm{D}_{1}$ & 56260 & $2.26[-2]$ & $2.23[-2]$ & $4.0[-2]$ & \\
\hline $5 s 5 p^{3} P_{1}^{0}-5 s 5 d^{3} D_{2}$ & 58824 & $1.86[+0]$ & $1.88[+0]$ & $1.89[+0]$ & \\
\hline $5 \mathrm{~s} 5 \mathrm{p}^{3} \mathrm{P}_{1}^{\mathrm{o}}-5 \mathrm{~s} 5 \mathrm{~d}^{3} \mathrm{D}_{1}$ & 58738 & $5.37[-1]$ & $5.40[-1]$ & $6.24[-1]$ & \\
\hline $5 \mathrm{~s} 5 \mathrm{p}^{3} \mathrm{P}_{0}^{\mathrm{o}}-5 \mathrm{~s} 5 \mathrm{~d}^{3} \mathrm{D}_{1}$ & 59813 & $8.77[-1]$ & $8.89[-1]$ & $8.55[-1]$ & \\
\hline $5 s 5 p^{3} P_{2}^{o}-5 s 6 d^{3} D_{3}$ & 79001 & $3.49[-1]$ & $3.63[-1]$ & $3.9[-1]$ & \\
\hline $5 s 5 p^{3} P_{2}^{o}-5 s 6 d^{3} D_{2}$ & 78947 & $6.29[-2]$ & $6.57[-2]$ & $7.10[-2]$ & \\
\hline $5 \mathrm{~s} 5 \mathrm{p}^{3} \mathrm{P}_{2}^{\mathrm{o}}-5 \mathrm{~s} 6 \mathrm{~d}^{3} \mathrm{D}_{1}$ & 78913 & $4.17[-3]$ & $4.37[-3]$ & $4.7[-3]$ & \\
\hline $5 \mathrm{~s} 5 \mathrm{p}^{3} \mathrm{P}_{1}^{\mathrm{o}}-5 \mathrm{~s} 6 \mathrm{~d}^{3} \mathrm{D}_{2}$ & 81426 & $1.91[-1]$ & $1.99[-1]$ & $2.17[-1]$ & \\
\hline $5 \mathrm{~s} 5 \mathrm{p}^{3} \mathrm{P}_{1}^{\mathrm{o}}-5 \mathrm{~s} 6 \mathrm{~d}^{3} \mathrm{D}_{1}$ & 81392 & $6.37[-2]$ & $6.65[-2]$ & $7.20[-2]$ & \\
\hline $5 \mathrm{~s} 5 \mathrm{p}^{3} \mathrm{P}_{0}^{\mathrm{o}}-5 \mathrm{~s} 6 \mathrm{~d}^{3} \mathrm{D}_{1}$ & 82467 & $8.60[-2]$ & $8.96[-2]$ & $9.8[-2]$ & \\
\hline $5 s 5 p^{3} P_{2}^{o}-5 s 7 d^{3} D_{3}$ & 88943 & $9.69[-2]$ & $1.04[-1]$ & $1.2[-1]$ & \\
\hline $5 s 5 p^{3} P_{2}^{o}-5 s 7 d^{3} D_{2}$ & 88915 & $1.72[-2]$ & $1.85[-2]$ & $2.2[-2]$ & \\
\hline $5 \mathrm{~s} 5 \mathrm{p}^{3} \mathrm{P}_{2}^{\mathrm{o}}-5 \mathrm{~s} 7 \mathrm{~d}^{3} \mathrm{D}_{1}$ & 88897 & $1.13[-3]$ & $1.22[-3]$ & $1.5[-3]$ & \\
\hline $5 \mathrm{~s} 5 \mathrm{p}^{3} \mathrm{P}_{1}^{\mathrm{o}}-5 \mathrm{~s} 7 \mathrm{~d}^{3} \mathrm{D}_{2}$ & 91393 & $5.41[-2]$ & $5.86[-2]$ & $6.93[-2]$ & \\
\hline $5 \mathrm{~s} 5 \mathrm{p}^{3} \mathrm{P}_{1}^{\mathrm{o}}-5 \mathrm{~s} 7 \mathrm{~d}^{3} \mathrm{D}_{1}$ & 91376 & $1.78[-2]$ & $1.93[-2]$ & $2.3[-2]$ & \\
\hline $5 \mathrm{~s} 5 \mathrm{p}^{3} \mathrm{P}_{0}^{\mathrm{o}}-5 \mathrm{~s} 7 \mathrm{~d}^{3} \mathrm{D}_{1}$ & 92450 & $2.43[-2]$ & $2.64[-2]$ & $3.10[-2]$ & \\
\hline
\end{tabular}

where $I$ is the nuclear spin and $F(=I)$ is the total angular momentum quantum number. The use of quotation marks in the left-hand wavefunction emphasizes the fact that the notation is just a label indicating the dominant character of the eigenvector. Clearly, the one-photon $5 \mathrm{~s} 5 \mathrm{p}^{3} \mathrm{P}_{0}^{\mathrm{o}} \rightarrow 5 \mathrm{~s}^{2}{ }^{1} \mathrm{~S}_{0}$ transition now becomes allowed via the mixing of functions with $J$ different from zero. $\left|5 \mathrm{~s} 5 \mathrm{p}^{3} \mathrm{P}_{1}^{\mathrm{o}} I F\right\rangle$ and $\left|5 \mathrm{~s} 5 \mathrm{p}^{1}{ }_{1}^{\mathrm{o}} I F\right\rangle$ are the only important contributors to wavefunction expansion and in this work we model the $5 \mathrm{~s} 5 \mathrm{p}^{3} \mathrm{P}_{0}^{\mathrm{o}}$ state as

$$
\left|{ }^{5} 5 \mathrm{~s} 5 \mathrm{p}^{3} \mathrm{P}_{0}^{\mathrm{o}} I F^{\prime}\right\rangle=c_{0}\left|5 \mathrm{~s} 5 \mathrm{p}^{3} \mathrm{P}_{0}^{\mathrm{o}} I F\right\rangle+c_{1}\left|5 \mathrm{~s} 5 \mathrm{p}^{3} \mathrm{P}_{1}^{\mathrm{o}} I F\right\rangle+c_{2}\left|5 \mathrm{~s} 5 \mathrm{p}^{1} \mathrm{P}_{1}^{\mathrm{o}} I F\right\rangle .
$$

The mixing coefficients are obtained by constructing and diagonalizing the Hamiltonian matrix in the presence of the hyperfine interaction. Once the mixing coefficients have been determined 
Table 5. Energies (in $\mathrm{cm}^{-1}$ ) and weighted oscillator strengths in Coulomb and Babushkin gauges for the $5 \mathrm{~s}^{2}{ }^{1} \mathrm{~S}_{0}-5 \mathrm{~s} 5 \mathrm{p}{ }^{3} \mathrm{P}_{1}^{\mathrm{o}}$ and $5 \mathrm{~s}^{2}{ }^{1} \mathrm{~S}_{0}-5 \mathrm{~s} 5 \mathrm{p}{ }^{1} \mathrm{P}_{1}^{\mathrm{o}}$ transitions from CI calculations with increasingly large configuration expansions. The first active set consists of the $5 \mathrm{~s}, 5 \mathrm{p}-, 5 \mathrm{p}, 5 \mathrm{~d}-, 5 \mathrm{~d}$ orbitals in the complex. The active set is enlarged by up to five layers of correlation orbitals.

\begin{tabular}{|c|c|c|c|c|c|c|}
\hline \multirow[b]{2}{*}{ Active set } & \multicolumn{3}{|c|}{$5 \mathrm{~s}^{2}{ }^{1} \mathrm{~S}_{0}-5 \mathrm{~s} 5 \mathrm{p}{ }^{1} \mathrm{P}_{1}^{\mathrm{o}}$} & \multicolumn{3}{|c|}{$5 \mathrm{~s}^{2}{ }^{1} \mathrm{~S}_{0}-5 \mathrm{~s} 5 \mathrm{p}^{3} \mathrm{P}_{1}^{\mathrm{o}}$} \\
\hline & $\Delta E$ & $g f_{B}$ & $g f_{C}$ & $\Delta E$ & $g f_{B}$ & $g f_{C}$ \\
\hline Complex & 64726 & 2.12 & 1.87 & 39753 & $2.12[-3]$ & $2.99[-3]$ \\
\hline Corr. 1 & 62953 & 1.65 & 1.65 & 43293 & $4.90[-3]$ & $5.37[-3]$ \\
\hline Corr. 2 & 62939 & 1.59 & 1.69 & 43427 & $5.16[-3]$ & $5.96[-3]$ \\
\hline Corr. 3 & 62806 & 1.58 & 1.71 & 43401 & $5.16[-3]$ & $5.99[-3]$ \\
\hline Corr. 4 & 62785 & 1.57 & 1.71 & 43387 & $5.14[-3]$ & $5.99[-3]$ \\
\hline Corr. 5 & 62773 & 1.57 & 1.71 & 43382 & $5.13[-3]$ & $5.99[-3]$ \\
\hline Spectrum & 62779 & 1.56 & 1.70 & 43141 & $4.91[-3]$ & $5.86[-3]$ \\
\hline $\mathrm{HFR}^{\mathrm{a}}$ & & 1.64 & & & $7.3[-3]$ & \\
\hline $\mathrm{HFR}^{\mathrm{b}}$ & & 1.522 & & & $5.19[-3]$ & \\
\hline $\mathrm{MCDHF}^{\mathrm{b}}$ & 62714 & 1.562 & 1.538 & 43655 & $4.71[-3]$ & $5.07[-3]$ \\
\hline Experiment & 63039 & $1.43(9)^{\mathrm{c}}$ & & 43351 & $5.44(45)[-3]^{\mathrm{d}}$ & \\
\hline
\end{tabular}

${ }^{\mathrm{a}}$ Biémont and Zeippen [15].

${ }^{\mathrm{b}}$ Biémont et al [16].

c Beam-foil (ANDC) (Ansbacher et al [7]).

${ }^{\mathrm{d}}$ Intensity modulated laser excitation (Peik et al [2]).

Table 6. Magnetic dipole, $A$, and electric quadrupole, $B$, hyperfine interaction constants (in Mhz) for $5 \mathrm{~s} 5 \mathrm{p}^{3} \mathrm{P}^{\mathrm{o}}$ in ${ }^{115} \mathrm{In}$ from calculations with increasingly large configuration expansions including single excitations from all core shells. The first active set consists of the $5 \mathrm{~s}, 5 \mathrm{p}-, 5 \mathrm{p}, 5 \mathrm{~d}-, 5 \mathrm{~d}$ orbitals in the complex. The active set is then enlarged by up to five layers of correlation orbitals.

\begin{tabular}{lllllll}
\hline Active set & $A\left({ }^{3} \mathrm{P}_{1}^{\mathrm{o}}\right)$ & $B\left({ }^{3} \mathrm{P}_{1}^{\mathrm{o}}\right)$ & $A\left({ }^{3} \mathrm{P}_{2}^{\mathrm{o}}\right)$ & $B\left({ }^{3} \mathrm{P}_{2}^{\mathrm{o}}\right)$ & $A\left({ }^{3} \mathrm{P}_{1}^{\mathrm{o}},{ }^{3} \mathrm{P}_{0}^{\mathrm{o}}\right)$ & $A\left({ }^{1} \mathrm{P}_{1}^{\mathrm{o}},{ }^{3} \mathrm{P}_{0}^{\mathrm{o}}\right)$ \\
\hline Complex & 5644 & -305 & 4362 & 540 & 5633 & 3733 \\
Corr. 1 & 6519 & -381 & 4814 & 688 & 6148 & 4152 \\
Corr. 2 & 6700 & -394 & 4916 & 715 & 6296 & 4141 \\
Corr. 3 & 6907 & -413 & 5056 & 750 & 6433 & 4258 \\
Corr. 4 & 6898 & -416 & 5046 & 753 & 6417 & 4237 \\
Corr. 5 & 6948 & -422 & 5082 & 765 & 6452 & 4270 \\
Spectrum & 6832 & -407 & 5014 & 737 & & \\
Experiment $^{\mathrm{a}}$ & $6961 \pm 6$ & $-477 \pm 27$ & $5093 \pm 12$ & $629 \pm 180$ & & \\
Experiment $^{\mathrm{b}}$ & $6949 \pm 24-420$ & $5088 \pm 27$ & 629 & & \\
\hline
\end{tabular}

${ }^{a}$ High-resolution scanning echelle monochromator (Larkins and Hannaford [6]).

${ }^{\mathrm{b}}$ Fourier transform spectra (Karlsson and Litzén [1]).

the transition rate can be computed as

$$
\begin{aligned}
A\left(5 \mathrm{~s} 5 \mathrm{p}^{3} \mathrm{P}_{0}^{\mathrm{o}} \rightarrow\right. & \left.5 \mathrm{~s}^{2}{ }^{1} \mathrm{~S}_{0}\right)=\frac{2.02613 \times 10^{18}}{3 \lambda^{3}} \mid c_{1}\left\langle 5 \mathrm{~s}^{2}{ }^{1} \mathrm{~S}_{0}\left\|\mathbf{Q}_{1}^{(1)}\right\| 5 \mathrm{~s} 5 \mathrm{p}^{3} \mathrm{P}_{1}^{\mathrm{o}}\right\rangle \\
& +c_{2}\left\langle\left. 5 \mathrm{~s}^{2}{ }^{1} \mathrm{~S}_{0}\left\|\mathbf{Q}_{1}^{(1)}\right\| 5 \mathrm{~s} 5 \mathrm{p}^{1} \mathrm{P}_{1}^{\mathrm{o}}\right|^{2}\right\rangle
\end{aligned}
$$

where the decay rate is in $\mathrm{s}^{-1}$ and $\lambda$ is the wavelength in $\AA$ for the transition. $\left\langle 5 \mathrm{~s}^{2}{ }^{1} \mathrm{~S}_{0}\left\|\mathbf{Q}_{1}^{(1)}\right\| 5 \mathrm{~s} 5 \mathrm{p}^{3} \mathrm{P}_{1}^{\mathrm{o}}\right\rangle$ and $\left\langle 5 \mathrm{~s}^{2}{ }^{1} \mathrm{~S}_{0}\left\|\mathbf{Q}_{1}^{(1)}\right\| 5 \mathrm{~s} 5 \mathrm{p}{ }^{1} \mathrm{P}_{1}^{\mathrm{o}}\right\rangle$ are reduced matrix elements for the electric dipole operator [5]. The reduced transition matrix elements can be obtained as square roots of the corresponding line strengths. The phase is such that we have constructive 
Table 7. Differences $\Delta S_{\mathrm{nms}}, \Delta S_{\mathrm{sms}}$ and $\Delta \rho(0)$ between the upper and lower state isotope shift parameters in the $5 \mathrm{~s}^{2}{ }^{1} \mathrm{~S}_{0}-5 \mathrm{~s} 5 \mathrm{p}^{3} \mathrm{P}_{1}^{\mathrm{o}}$ transition from CI calculations with increasingly large configuration expansions. The first active set consists of the $5 \mathrm{~s}, 5 \mathrm{p}-, 5 \mathrm{p}, 5 \mathrm{~d}-, 5 \mathrm{~d}$ orbitals in the complex. The active set is enlarged by up to five layers of correlation orbitals.

\begin{tabular}{lcrc}
\hline Active set & $\Delta S_{\text {sms }}$ & $\Delta \rho(0)$ & $\Delta S_{\mathrm{nms}}$ \\
\hline Complex & -0.40131 & -26.170 & -0.10488 \\
Corr. 1 & -0.58762 & -30.032 & -0.16504 \\
Corr. 2 & -0.59522 & -29.951 & -0.14131 \\
Corr. 3 & -0.56657 & -30.378 & -0.18993 \\
Corr. 4 & -0.56183 & -30.213 & -0.19124 \\
Corr. 5 & -0.56125 & -30.279 & -0.19219 \\
\hline
\end{tabular}

Table 8. Computed and experimental transition isotope shift $\Delta v=v_{115}-v_{113}$ (in $\mathrm{GHz}$ ) for $5 \mathrm{~s}^{2}{ }^{1} \mathrm{~S}_{0}-5 \mathrm{~s} 5 \mathrm{p}^{3} \mathrm{P}_{1}^{\mathrm{o}}$.

\begin{tabular}{llrl}
\hline$\Delta v_{\mathrm{nms}}$ & $\Delta v_{\mathrm{sms}}$ & $\Delta v_{\mathrm{vol}}$ & \multicolumn{1}{c}{$\Delta v_{\mathrm{tot}}$} \\
\hline 0.107 & 0.312 & -0.935 & -0.515 \\
& & & $-0.64(7)^{\mathrm{a}}$ \\
\hline
\end{tabular}

a Peik et al [2].

interference. To obtain a good value for the rate, both the hyperfine interaction and the transition matrix elements must be calculated with high accuracy. The off-diagonal magnetic hyperfine interaction constants needed to construct the Hamiltonian matrix elements are $A\left({ }^{3} \mathrm{P}_{1}^{\mathrm{o}},{ }^{3} \mathrm{P}_{0}^{\mathrm{o}}\right)=3928 \mathrm{MHz}$ and $A\left({ }^{1} \mathrm{P}_{1}^{\mathrm{o}},{ }^{3} \mathrm{P}_{0}^{\mathrm{o}}\right)=2916 \mathrm{MHz}$. The reader may consult [29] for explicit formulae relating the interaction constants and the matrix elements. The offdiagonal magnetic hyperfine interaction constants give mixing coefficients $c_{1}=1.02 \times 10^{-3}$ and $c_{2}=3.47 \times 10^{-5}$ in good agreement with the values $c_{1}=1.1 \times 10^{-3}$ and $c_{2}=3.5 \times 10^{-5}$ derived by Peik et al from the Breit-Wills theory for a hyperfine structure in intermediate coupling. Using the transition matrix elements $\left\langle 5 \mathrm{~s}^{2}{ }^{1} \mathrm{~S}_{0}\left\|\mathbf{Q}_{1}^{(1)}\right\| 5 \mathrm{~s} 5 \mathrm{p}^{3} \mathrm{P}_{1}^{\mathrm{o}}\right\rangle=3.89 \times 10^{-2}$ and $\left\langle 5 \mathrm{~s}^{2}{ }^{1} \mathrm{~S}_{0}\left\|\mathbf{Q}_{1}^{(1)}\right\| 5 \mathrm{~s} 5 \mathrm{p}^{1} \mathrm{P}_{1}^{\mathrm{o}}\right\rangle=8.21$ from the calculations reported in table 5 we obtain a transition rate $A=4.61 \mathrm{~s}^{-1}$ corresponding to a lifetime $\tau=0.217 \mathrm{~s}$ of the ${ }^{3} \mathrm{P}_{0}^{\mathrm{o}}$ state. This value should be compared with the experimental value $\tau=0.14(2) \mathrm{s}$ determined by Peik et al [2]. One explanation for the difference between theory and experiment may be that the current calculation underestimates the transition matrix element for the intercombination transition. Another explanation could be that the neglected two-photon decay channel may be important. More work, both theoretical and experimental, is needed to clarify the situation.

\section{Summary and conclusions}

We report on relativistic multiconfiguration Dirac-Hartree-Fock calculations of oscillator strengths for electric dipole transitions in In II. In addition hyperfine structure constants and Landé $g_{J}$-factors are given. On the whole the calculations, that account for core polarization by means of explicit CI, give a good description of the energy structure. The calculated hyperfine magnetic dipole interaction constants are in excellent agreement with constants derived from Fourier transform spectra. For the electric quadrupole interaction constants the situation is different. Here there are substantial differences especially for the $5 \mathrm{~s} n \mathrm{~d}^{3} \mathrm{D}$ states. These differences indicate that parameterizations using only diagonal interaction constants are 
inadequate and that accurate descriptions of the experimental, partly resolved, line profiles must include also off-diagonal contributions [22].

Looking at the oscillator strengths there is good agreement between the results in the Coulomb and Babushkin gauges. The oscillator strengths from the spectrum calculations were compared with more accurate calculations for the $5 \mathrm{~s}^{2}{ }^{1} \mathrm{~S}_{0}-5 \mathrm{~s} 5 \mathrm{p}^{3} \mathrm{P}_{1}^{\mathrm{o}}$ and $5 \mathrm{~s}^{2}{ }^{1} \mathrm{~S}_{0}-5 \mathrm{~s} 5 \mathrm{p}{ }^{1} \mathrm{P}_{1}^{\mathrm{o}}$ transitions where the correlation orbitals were targeted only on the states in the transition. The agreement between the two different sets of calculations is good, showing that the major correlation effects are captured in the spectrum calculations.

The calculated ${ }^{115-113}$ In isotope shift for the $5 \mathrm{~s}^{2}{ }^{1} \mathrm{~S}_{0}-5 \mathrm{~s} 5 \mathrm{p}^{3} \mathrm{P}_{1}^{\mathrm{o}}$ transition is within the experimental error bars. The major uncertainty is the specific mass shift contribution which is very difficult to obtain accurately. Using calculated off-diagonal hyperfine interaction constants and electric dipole transition matrix elements the lifetime for the $5 \mathrm{~s} 5 \mathrm{p}^{3} \mathrm{P}_{0}^{\mathrm{o}}$ state was determined to $0.217 \mathrm{~s}$. The computed lifetime is considerably longer that the experimental lifetime of 0.14(2) s, and the difference may be due to underestimated transition matrix elements.

\section{Acknowledgment}

Financial support by the Swedish Research Council is gratefully acknowledged.

\section{References}

[1] Karlsson H and Litzén U 2001 J. Phys. B: At. Mol. Opt. Phys. 344475

[2] Peik E, Hollemann G and Walther H 1994 Phys. Rev. A 49402

[3] Indelicato P, Parente F and Marrus R 1989 Phys. Rev. A 403505

[4] Aboussaïd A, Godefroid M R, Jönsson P and Froese Fischer C 1995 Phys. Rev. A 512031

[5] Johnson W R, Cheng K T and Plante D R 1997 Phys. Rev. A 552728

[6] Larkins P L and Hannaford P 1993 Z. Phys. D 27313

[7] Ansbacher W, Pinnington E H, Kernahan J A and Gosselin R N 1986 Can. J. Phys. 641365

[8] Hibbert A 1982 Nucl. Instrum. Methods 202323

[9] Migdalek J and Baylis W E 1986 J. Phys. B: At. Mol. Phys. 191

[10] Migdalek J and Bojara A 1988 J. Phys. B: At. Mol. Opt. Phys. 212221

[11] Das B P and Idrees M 1990 Phys. Rev. A 426900

[12] Chou H-S and Huang K-N 1992 Phys. Rev. A 463725

[13] Chou H-S, Chi H-C and Huang K-N 1993 Phys. Rev. A 482453

[14] Lavin C and Martin I 1994 J. Quant. Spectrosc. Radiat. Transfer 5221

[15] Biémont E and Zeippen C J 1999 At. Data Nucl. Data Tables 72101

[16] Biémont E, Froese Fischer C, Godefroid M R, Palmeri P and Quinet P 2000 Phys. Rev. A 62032512

[17] Asplund M, Grevesse N and Sauval A J 2005 Cosmic Abundances as Records of Stellar Evolution and Nucleosynthesis in Honor of David L Lambert (ASP Conference Series vol 336) ed T G Barnes III and F N Bash p 25

[18] Jönsson P, He X, Froese Fischer C and Grant I P 2007 Comput. Phys. Commun. submitted

[19] Grant I P 2007 Relativistic Quantum Theory of Atoms and Molecules: Theory and Computation (Springer Series on Atomic, Optical, and Plasma Physics) (Berlin: Springer)

[20] McKenzie B J, Grant I P and Norrington P H 1980 Comput. Phys. Commun. 21233

[21] Jönsson P and Froese Fischer C 1997 Comput. Phys. Commun. 10081

[22] Andersson M, Jönsson P and Sabel H 2006 J. Phys. B: At. Mol. Opt. Phys. 394239

[23] Jönsson P, Parpia F A and Froese Fischer C 1996 Comput. Phys. Commun. 96301

[24] Andersson M and Jönsson P 2007 Comput. Phys. Commun. at press

[25] Stone N J 2005 At. Data Nucl. Data Tables 9075

[26] Grant I P 1974 J. Phys. B: At. Mol. Opt. Phys. 71458

[27] Olsen J, Godefroid M, Jönsson P, Malmqvist P Å and Froese Fischer C 1995 Phys. Rev. E 524499

[28] Dyall K G, Grant I P, Johnson C T, Parpia F A and Plummer E-P 1989 Comput. Phys. Commun. 55425 
[29] Froese Fischer C, Brage T and Jönsson P 1997 Computational Atomic Structure-An MCHF Approach (Bristol: Institute of Physics Publishing)

[30] Karlsson H 2002 Spectroscopic Studies of the Elements Ba, Fe, Ga, In and Ru (Lund University, Sweden: Atomic Astrophysics Lund Observatory)

[31] Andersson M, Bieroń J and Jönsson P 2007 in preparation

[32] Eberz J et al 1987 Nucl. Phys. A 4649 